\title{
ON QUASI-ELLIPTIC BOUNDARY PROBLEMS
}

\author{
BY \\ TADATO MATSUZAWA
}

1. Introduction. In this paper we consider the boundary problem

$$
\begin{gathered}
P(x, D) u=f \quad \text { in } \quad x_{n}>0, \\
Q_{j}(x, D) u=g_{j} \quad \text { on } \quad x_{n}=0,1 \leqq j \leqq r,
\end{gathered}
$$

where $P(x, D)$ is a quasi-elliptic operator and $\left\{Q_{j}(x, D)\right\}_{j=1}^{r}$ is a system of boundary operators satisfying the complementing condition.

First we derive the a priori estimate of the form

$$
\|v\|_{m} \leqq C\left(\|P(x, D) v\|_{0}+\sum_{j=1}^{r}\left|Q_{j}(x, D) v\right|_{k_{j}}+\|v\|_{0}\right)
$$

for suitable boundary norms $\left|Q_{j}(x, D) v\right|_{k,}, j=1, \ldots, r$.

Secondly we shall consider about the hypo-analyticity at the boundary $x_{n}=0$ for the above problem with the simple boundary operators.

In $\$ 2$, a definition of a quasi-elliptic operator is given. In $\$ 3$, a definition of a quasi-elliptic boundary problem is given. $\$ 4$ and $\$ 5$ are devoted to derive an a priori estimate (coerciveness estimate) for the case of constant coefficients. In $\$ 6$ and $\$ 7$, we shall prove the coerciveness estimate for the case of variable coefficients. In $\$ 8$ we prove the regularity at the boundary of the solutions of quasielliptic boundary problem.

In $\$ 9,10$ and 11 we consider hypo-analyticity at the boundary for quasielliptic boundary problems. Theorem 9.2 was suggested by Professor Lions.

The author is deeply indebted to the authors [2], [3] and [12] to make this paper. The author expresses his hearty thanks to Professors Mizohata, Lions, and Kuroda for their valuable suggestions.

2. Preliminaries. Let $R^{n}$ be the $n$-dimensional Euclidean space whose point is denoted by $\left(x_{1}, \ldots, x_{n}\right)$. For convenience, set $x=\left(x_{1}, \ldots, x_{n-1}\right), y=x_{n}$ and denote by $(x, y)$ a point of $R^{n}$. The half spaces $y>0$ and $y \geqq 0$ are denoted by $R_{+}^{n}$ and $\left(R_{+}^{n}\right)^{a}$ respectively.

Let $\alpha=\left(\alpha_{1}, \ldots, \alpha_{n}\right)$ be a multi-index of nonnegative integers with length $|\alpha|=\alpha_{1}+\cdots+\alpha_{n}$. We put $D_{j}=i^{-1}\left(\partial / \partial x_{j}\right), 1 \leqq j \leqq n,\left(i^{2}=-1\right)$, and

$$
D_{x}=\left(D_{1}, \ldots, D_{n-1}\right), \quad D_{y}=D_{n}, \quad D=\left(D_{1}, \ldots, D_{n}\right) .
$$

Received by the editors April 6, 1967 and, in revised form, June 20, 1967. 
Then the linear differential operators of order $m$ with constant coefficients can be written as

$$
P(D)=P\left(D_{x}, D_{y}\right)=\sum_{|\alpha| \leqq m} a_{\alpha} D_{1}^{\alpha} \cdots D_{n^{n}}^{\alpha},
$$

where the coefficients $a_{\alpha}$ are complex constants. The polynomial corresponding to $P\left(D_{x}, D_{y}\right)$ is

$$
P(\xi, \eta)=\sum_{|\alpha| \leqq m} a_{\alpha} \xi_{1}^{\alpha} \cdots \xi_{n-1}^{\alpha_{n}-1} \eta^{\alpha_{n}}
$$

where $\xi=\left(\xi_{1}, \ldots, \xi_{n-1}\right)$.

Definition 2.1. A quasi-elliptic operator of weight $q$ is defined as an operator (2.1) satisfying the following conditions:

$$
\begin{gathered}
m_{j}>0, \quad 1 \leqq i \leqq n, \quad \text { are given integers, } m=\max _{1 \leqq j \leqq n} m_{j}, \\
\quad q_{j}=m / m_{j} \geqq 1, \text { and } q=\left(q_{1}, \ldots, q_{n}\right) . \\
P(D)=\sum_{\langle\alpha, q\rangle \leqq m} a_{\alpha} D^{\alpha}, \text { where }\langle\alpha, q\rangle=\alpha_{1} q_{1}+\cdots+\alpha_{n} q_{n}
\end{gathered}
$$

the principal part of $P$ with respect to the weight $q$ is $P^{0}(D)=\sum_{\langle\alpha, q\rangle=m} a_{\alpha} D^{\alpha}$.

$$
\sum_{1 \leqq j \leqq n-1}\left|\xi_{j}\right|^{m_{j}}+|\eta|^{m_{n}} \leqq C\left|P^{0}(\xi, \eta)\right| \text { for any }(\xi, \eta) \in R^{n}
$$

We see that the quasi-elliptic operators are hypo-elliptic. We note that when $m_{j}=m$ for every $j$, the quasi-elliptic operators are just the elliptic operators of order $m$. If $m_{n}=1$ and $m_{j}=2$ for $j<n$, we find that the heat equation is quasielliptic. Also the $p$-parabolic equations in the sense of Petrowsky are quasi-elliptic (cf. Friberg [3]).

Let $\tau_{1}(\xi), \ldots, \tau_{m_{n}}(\xi)$ denote the roots of $P^{0}(\xi, z)=0$ for each real vector $\xi=$ $\left(\xi_{1}, \ldots, \xi_{n-1}\right)$. In the case $n>2$ we see from the condition (2.4) that the number $r$ of the roots with the positive imaginary part is independent of $\xi \neq 0$, and in this case we shall say that $P(\xi, \eta)(P(D))$ is of determined type $r$. In the case of $n=2$ we suppose this root-condition on $P^{0}(\xi, \eta)$.

3. Coerciveness inequality (I). The constant coefficient case.

We consider a quasi-elliptic operator $P(D)$ of weight $q=\left(m / m_{1}, \ldots, m / m_{n}\right)$ and of determined type $r\left(1 \leqq r \leqq m_{n}\right)$ :

$$
P(D)=P\left(D_{x}, D_{y}\right)=\sum_{\langle\alpha, q\rangle \leq m} a_{\alpha} D_{1}^{\alpha} \cdots D_{n-1}^{\alpha_{n}-1} D_{n}^{\alpha}
$$

Here we may assume the coefficient of $D_{n}^{m}$ is equal to 1 . The corresponding polynomial of $P(D)$ is

$$
P(\xi, \eta)=\sum_{\langle\alpha, q\rangle \leqq m} a_{\alpha} \xi_{1}^{\alpha_{1}} \cdots \xi_{n-1}^{\alpha_{n}-1} \eta^{\alpha_{n}}
$$


By rearrangement if necessary we may assume that

$$
\begin{array}{ll}
\operatorname{Im} \tau_{k}(\xi)>0, & 1 \leqq k \leqq r, \\
\operatorname{Im} \tau_{k}(\xi)<0, & r<k \leqq m_{n},
\end{array}
$$

Set

$$
P_{+}=\prod_{k=1}^{r}\left(\eta-\tau_{k}(\xi)\right), \quad P_{-}=P^{0} / P_{+} .
$$

Similarly we consider the boundary operators of the form

$$
Q_{j}(D)=\sum_{\langle\beta, q\rangle \leqq p_{j}} b_{\beta} D^{\beta}, \quad j=1, \ldots, r, \quad 0 \leqq p_{j} \leqq\left(m_{n}-1\right) \frac{m}{m_{n}}=m-q_{n} .
$$

The corresponding polynomial of $Q_{j}(D)$ is

$$
Q_{j}(\xi, \eta)=\sum_{\langle\beta, q\rangle \leq p_{j}} b_{\beta} \xi_{1}^{\beta_{1}} \cdots \xi_{n-1}^{\beta_{n}-1} \eta^{\beta_{n}}
$$

and

$$
Q_{j}^{0}(\xi, \eta)=\sum_{\langle\beta, q\rangle=p_{j}} b_{\beta} \xi_{1}^{\beta_{1}} \cdots \xi_{n}^{\beta_{n}-1} \eta^{\beta_{n}}
$$

Definition 3.1 (Complementing Condition). We shall say that the $Q_{j}(D)$ $(j=1, \ldots, r)$ cover $P(D)$ when $Q_{j}^{0}(\xi, \eta)(j=1, \ldots, r)$ are linearly independent modulo $P_{+}(\xi, \eta)$ as the polynomials in $\eta$ for every nonzero $\xi \in R^{n-1}$.

Let $C_{0}\left(R_{+}^{n}\right)$ denote the set of complex-valued functions which are infinitely differentiable in $R_{+}^{n}$ and vanish for $(x, y)$ with $|x|^{2}+y^{2}$ sufficiently large. We denote by $\hat{v}(\xi, y)$ the Fourier transform of $v(x, y) \in C_{0}^{\infty}\left(\left(R_{+}^{n}\right)^{-}\right)$with respect to the variables $x_{1}, \ldots, x_{n-1}$ :

$$
\hat{v}(\xi, y)=(2 \pi)^{-(n-1) / 2} \int_{R^{n-1}} e^{-i\langle\xi, x\rangle} v(x, y) d x .
$$

As usual we set

$$
\|v\|=\left(\iint_{\left(R_{+}^{n}\right)^{a}}|v(\dot{x}, y)|^{2} d x d y\right)^{1 / 2}=\left(\int_{0}^{\infty} \int_{|\xi|<\infty}|\hat{v}(\xi, y)|^{2} d \xi d y\right)^{1 / 2} .
$$

Corresponding to the operator (3.1) we employ the notation

$$
\langle\xi\rangle=\left(\left|\xi_{1}\right|^{m_{1}}+\cdots+\left|\xi_{n-1}\right|^{m_{n-1}}\right)^{1 / m} \text {. }
$$

For a real number $p$, we shall make use of the scalar product

$$
\left\langle v_{1}, v_{2}\right\rangle_{p}=\int_{R^{n-1}}\left(1+\langle\xi\rangle^{2}\right)^{p} \hat{v}_{1}(\xi, 0) \overline{\hat{v}_{2}(\xi, 0)} d \xi, v_{1}, v_{2} \in C_{0}^{\infty}\left(\left(R_{+}^{n}\right)^{a}\right) .
$$

The corresponding norm is given by

$$
|v|_{p}=\left(\langle v, v\rangle_{p}\right)^{1 / 2}
$$

Then we have the following two theorems. 
THEOREM 3.1. Let $P(D)$ and $Q_{j}(D), j=1, \ldots, r$, be given as above and $Q_{f}(D)(j=1, \ldots, r)$ cover $P(D)$. Then there is a constant $\left(^{1}\right) C$ depending only on $P^{0}$ and $Q_{j}^{0}(j=1, \ldots, r)$ such that

$$
\sum_{\langle\alpha, q\rangle=m}\left\|D^{\alpha} v\right\| \leqq C\left(\left\|P^{0}(D) v\right\|+\sum_{j=1}^{r}\left|Q_{j}^{0}(D) v\right|_{m-p_{j}-\left(m / 2 m_{n}\right)}\right)
$$

for all $v \in C_{0}^{\infty}\left(\left(R_{n}^{+}\right)^{-}\right)$.

THEOREM 3.2. Let $P(D)$ and $Q_{j}(D), j=1, \ldots, r$, be the same as in Theorem 3.1. Then there is a constant $C$ depending only on $P$ and $Q_{j}(j=1, \ldots, r)$ such that

$$
\sum_{\langle\alpha, q\rangle \leqq m}\left\|D^{\alpha} v\right\| \leqq C\left(\|P(D) v\|+\sum_{j=1}^{r}\left|Q_{j}(D) v\right|_{m-p_{j}-\left(m / 2 m_{n}\right)}+\|v\|\right)
$$

for all $v \in C_{0}^{\infty}\left(\left(R_{+}^{n}\right)^{a}\right)$.

Theorem 3.1 can be proved by the modification of Schechter's method [12], we shall give the complete proof in the following section. The proof of Theorem 3.2 will be given in $\S 5$.

4. Proof of Theorem 3.1. Let $R(\xi, \eta)$ be any monomial such that

$$
R(\xi, \eta)=\xi_{1}^{\alpha_{1}} \cdots \xi_{n-1}^{\alpha_{n}-1} \eta^{\alpha_{n}}, \quad\langle\alpha, q\rangle=m .
$$

Then there is a constant $K_{1}$ such that

$$
|R(\xi, \eta)| \leqq K_{1}\left|P^{0}(\xi, \eta)\right|, \quad(\xi, \eta) \in R^{n} .
$$

In fact, $R(\xi, \eta) / P^{0}(\xi, \eta)$ is continuous on the surface $|\xi|^{2}+\eta^{2}=1$ in $R^{n}$, and to replace $\xi_{j}$ by $\xi_{j} t^{1 / m_{j}}, j=1, \ldots, n-1$ and $\eta$ by $\eta t^{1 / m_{n}}$ in (4.2) is nothing but to multiply both sides of (4.2) by $t$ if $t>0$. Hence (4.2) is valid for every $(\xi, \eta) \in R^{n}$.

Now it will be convenient to make the following definition.

Definition 4.1. We shall say that a function $p(\xi, \eta)$ is homogeneous of degree $k$ (with respect to weight $q$ ), if for any $t>0$ it holds

$$
p\left(t^{m / m_{1}} \xi_{1}, t^{m / m_{2}} \xi, \ldots, t^{m / m_{n}} \eta\right)=t^{k} p(\xi, \eta), \quad(\xi, \eta) \in R^{n} .
$$

We recall that $Q_{j}^{0}(\xi, \eta)=\sum_{\langle\alpha, q\rangle=p}, b_{\alpha} \xi_{1}^{\alpha_{1}} \cdots \xi_{n-1}^{\alpha_{n} 1} \eta^{\alpha_{n}}, 0 \leqq p_{j} \leqq m-\left(m / m_{n}\right)$ that is, $Q_{j}^{0}$ is homogeneous of degree $p_{j}$ with respect to weight $q$. We observe that by multiplying each $Q_{j}^{0}(\xi, \eta)$ by an appropriate power of $\langle\xi\rangle=\left(\left|\xi_{1}\right|^{m_{1}}+\cdots+\left|\xi_{n-1}\right|^{m_{n-1}}\right)^{1 / m}$, we may assume that each $Q_{j}^{0}(\xi, \eta)$ is homogeneous of degree $m-\left(m / m_{n}\right)$ with respect to weight $q$. Then $Q_{j}^{0}(\xi, \eta), 1 \leqq j \leqq r$, may no longer be polynomials in the $\xi$, but this does not affect the following argument.

For simplicity we assume that the roots $\tau_{k}(\xi)$ of $P^{0}(\xi, z)=0$ are simple, because

(1) In this paper we use the same symbol $C$ to express different constants. 
it is easy to prove Theorem 3.1 for the general case. Resolving into partial fractions we have

$$
\frac{R}{P^{0}}=\sum_{k=1}^{m_{n}} \frac{e_{k}}{\eta-\tau_{k}}, \quad \frac{Q_{j}^{0}}{P^{0}}=\sum_{k=1}^{m_{n}} \frac{q_{j k}}{\eta-\tau_{k}},
$$

where

$$
e_{k}(\xi)=\frac{R\left(\xi, \tau_{k}\right)}{\frac{\partial P^{0}\left(\xi, \tau_{k}\right)}{\partial \eta}}, \quad q_{j k}(\xi)=\frac{Q_{j}^{0}\left(\xi, \tau_{k}\right)}{\frac{\partial P^{0}\left(\xi, \tau_{k}\right)}{\partial \eta}}, \quad 1 \leqq j \leqq r, 1 \leqq k \leqq m_{n}
$$

We can easily verify

$$
\begin{array}{ll}
t^{m / m_{n}} \tau_{k}(\xi)=\tau_{k}\left(t^{m / m_{1}} \xi_{1}, \ldots, t^{m / m_{n-1}} \xi_{n-1}\right), & t>0,1 \leqq k \leqq m_{n}, \\
t^{m / m_{n}} e_{k}(\xi)=e_{k}\left(t^{m / m_{1}} \xi_{1}, \ldots, t^{m / m_{n-1}} \xi_{n-1}\right), & t>0,1 \leqq k \leqq m_{n} .
\end{array}
$$

Similarly we have

(4.6) $q_{j k}(\xi)=q_{j k}\left(t^{m / m_{1}} \xi_{1}, \ldots, t^{m / m_{n-1}} \xi_{n-1}\right), \quad t>0,1 \leqq j \leqq r, 1 \leqq k \leqq m_{n}$.

In particular, it follows that there are constants $K_{2}$ and $K_{3}$ such that

$$
\begin{aligned}
\left|e_{k}(\xi)\right| \leqq K_{2}\langle\xi\rangle^{m / m_{n}}=K_{2}\left(\sum_{j=1}^{n-1}\left|\xi_{j}\right|^{m_{j}}\right)^{1 / m_{n}}, & 1 \leqq k \leqq m_{n}, \\
K_{3}^{-1}\langle\xi\rangle^{m / m_{n}} \leqq\left|\operatorname{Im} \tau_{k}(\xi)\right| \leqq K_{3}\langle\xi\rangle^{m / m_{n}}, & 1 \leqq k \leqq m_{n} .
\end{aligned}
$$

Let $\hat{v}(\xi, y)$ be the Fourier transform of $v(x, y) \in C_{0}^{\infty}\left(\left(R_{+}^{n}\right)^{a}\right)$ with respect to the variables $x_{1}, \ldots, x_{n-1}$ and define it to be zero for $y<0$. We consider $\hat{v}(\xi, y)$ as a function of $y$ with a vector parameter $\xi$. Set

$$
\hat{v}(\xi, \eta)=\frac{1}{(2 \pi)^{1 / 2}} \int_{-\infty}^{\infty} e^{-i \eta \cdot y} \hat{v}(\xi, y) d y .
$$

Then we see that [recall that $D_{y}=i^{-1}(\partial / \partial y)$ ]

$$
\left[D_{y} v\right]^{\sim}=\eta \hat{v}+\frac{i}{(2 \pi)^{1 / 2}} \hat{v}(\xi, 0) .
$$

Next define

$$
\begin{aligned}
f(\xi, y) & =P^{0}\left(\xi, D_{y}\right) \hat{v}(\xi, y) & & \text { for } y \geqq 0 \\
& =0 & & \text { for } y<0,
\end{aligned}
$$

and set

$$
P_{k}^{0}(\xi, \eta)=\frac{P^{0}(\xi, \eta)}{\eta-\tau_{k}(\xi)}, \quad 1 \leqq k \leqq m_{n}
$$

Then by (4.9)

$$
f^{x}=\left(\eta-\tau_{k}\right)\left[P_{k}^{0}\left(\xi, D_{y}\right) \hat{v}\right]^{\sim}+W_{k}, \quad 1 \leqq k \leqq m_{n},
$$


where

$$
W_{k}=\frac{i}{(2 \pi)^{1 / 2}} P_{k}^{0}\left(\xi, D_{y}\right) \hat{v}(\xi, 0), \quad 1 \leqq k \leqq m_{n}
$$

Since

$$
R=\sum_{k=1}^{m_{n}} e_{k} P_{k}^{0}
$$

we have by (4.9)

$$
\begin{aligned}
{\left[R\left(\xi, D_{y}\right) \hat{v}\right]^{\sim} } & =\sum_{k=1}^{m_{n}} e_{k}\left[P_{k}^{0}\left(\xi, D_{y}\right) \hat{v}\right]^{\sim} \\
& =\sum_{k=1}^{m_{n}} e_{k} \frac{f-W_{k}}{\eta-\tau_{k}} \\
& =\frac{R}{P^{0}} \tilde{f}-\sum_{k=1}^{m_{n}} \frac{e_{k} W_{k}}{\eta-\tau_{k}}
\end{aligned}
$$

and hence

$$
\left|\left[R\left(\xi, D_{y}\right) \hat{v}\right]^{\sim}\right| \leqq K_{1}|\tilde{f}|+K_{2}\langle\xi\rangle^{m / m_{n}} \sum_{k=1}^{m_{n}} \frac{\left|W_{k}\right|}{\left|\eta-\tau_{k}\right|} .
$$

Thus by Parseval's formula and (4.8) we see

$$
\int_{0}^{\infty}\left|R\left(\xi, D_{y}\right) \hat{v}\right|^{2} d y \leqq C\left(\int_{-\infty}^{\infty}\left|f^{2}\right|^{2} d \eta+\langle\xi\rangle^{2 m / m_{n}} \sum_{k=1}^{m_{n}}\left|W_{k}\right|^{2} \int_{-\infty}^{\infty} \frac{d \eta}{\left|\eta-\tau_{k}\right|^{2}}\right)
$$

$$
\leqq C^{\prime}\left(\int_{-\infty}^{\infty}|\tilde{f}|^{2} d \eta+\langle\xi\rangle^{m / m_{n}} \sum_{k=1}^{m_{n}}\left|W_{k}\right|^{2}\right) .
$$

We note that Im $\tau_{k}<0$ for $r<k \leqq m_{n}$. Paley-Wiener's theorem implies

$$
\int_{-\infty}^{\infty} \frac{f}{\eta-\tau_{k}} d \eta=-2 \pi i W_{k}, \quad r<k \leqq m_{n} .
$$

Hence by (4.8) and by Schwarz's inequality

$$
\left|W_{k}\right|^{2} \leqq \text { const. }\langle\xi\rangle^{-m / m_{n}} \int_{-\infty}^{\infty}|\tilde{f}|^{2} d \eta, \quad r<k \leqq m_{n} .
$$

Therefore (4.11) becomes

$$
\int_{0}^{\infty}\left|R\left(\xi, D_{y}\right) \hat{v}\right|^{2} d y \leqq C\left(\int_{-\infty}^{\infty}|\tilde{f}|^{2} d \eta+\langle\xi\rangle^{m / m_{n}} \sum_{k=1}^{r}\left|W_{k}\right|^{2}\right) .
$$

Next we observe that there is no complex vector $\omega=\left(\omega_{1}, \ldots, \omega_{r}\right) \neq 0$ such that $\sum_{k=1}^{r} q_{j k} \omega_{k}=0,1 \leqq k \leqq r$. For, otherwise there would be a complex vector $\lambda=\left(\lambda_{1}, \ldots, \lambda_{r}\right) \neq 0$ such that

$$
\sum_{j=1}^{r} \lambda_{j} q_{j k}=0, \quad 1 \leqq k \leqq r,
$$


and hence

$$
P^{0-1} \sum_{j=1}^{r} \lambda_{j} Q_{j}^{0}=\sum_{j=1}^{r} \sum_{k=1}^{m_{n}} \frac{\lambda_{j} q_{j k}}{\eta-\tau_{k}}=\sum_{k=r+1}^{m_{n}} \sum_{j=1}^{r} \frac{\lambda_{j} q_{j k}}{\eta-\tau_{k}}
$$

which shows that $\sum_{j=1}^{r} \lambda_{j} Q_{j}^{0}$ is a multiple of $P_{+}$. This contradicts the complementing condition (Definition 3.1). Thus the expression

$$
\sum_{j=1}^{r}\left|\sum_{k=1}^{r} q_{j k} \omega_{k}\right|^{2}
$$

is positive on the compact set $\sum_{k=1}^{r}\left|\omega_{k}\right|^{2}=1,|\xi|=1$. Hence by the homogeneity properties of the $q_{j k}$, there exists some constant $K_{4}$

$$
\sum_{k=1}^{r}\left|\omega_{k}\right|^{2} \leqq K_{4} \sum_{j=1}^{r}\left|\sum_{k=1}^{r} q_{j k} \omega_{k}\right|^{2}
$$

for all $\omega$ and $\xi$.

Now recall that

$$
\begin{aligned}
Q_{j}^{0} & =\sum_{k=1}^{r} q_{j k} P_{k}^{0}+\sum_{k=r+1}^{m_{n}} q_{j k} P_{k}^{0}, \\
W_{k} & =\frac{i}{(2 \pi)^{1 / 2}} P_{k}^{0}\left(\xi, D_{y}\right) \hat{v}(\xi, 0) .
\end{aligned}
$$

By the triangle inequality

$$
\left|\sum_{k=1}^{r} q_{j k} P_{k}^{0} \hat{v}(\xi, 0)\right| \leqq\left|Q_{j}^{0} \hat{v}(\xi, 0)\right|+\left|\sum_{k=r+1}^{m_{n}} q_{j k} W_{k}\right|(2 \pi)^{1 / 2} .
$$

Hence combining (4.12), (4.13) and (4.15) we get

$$
\int_{0}^{\infty}\left|R\left(\xi, D_{y}\right) \hat{v}(\xi, y)\right|^{2} d y \leqq C\left(\int_{-\infty}^{\infty}|\tilde{f}|^{2} d \eta+(1+\langle\xi\rangle)^{m / m_{n}} \sum_{j=1}^{r}\left|Q_{j}^{0} \hat{v}(\xi, 0)\right|^{2}\right) .
$$

If we now integrate with respect to $\xi$ we obtain (3.7). This completes the proof.

5. Proof of Theorem 3.2. First we need the following lemma.

Lemma 5.1. For any $\varepsilon>0$ there exists a constant $C=C(\varepsilon)>0$ such that

$$
\sum_{\langle\beta, q\rangle<m}\left\|D^{\beta} v\right\| \leqq \varepsilon \sum_{\langle\alpha, q\rangle=m}\left\|D^{\alpha} v\right\|+C\|v\|, \quad v \in C_{0}^{\infty}\left(\left(R_{+}^{n}\right)^{a}\right) .
$$

Proof. We extend $v=v(x, y) \in C_{0}^{\infty}\left(\left(R_{+}^{n}\right)^{a}\right)$ to the whole space $R^{n}$ setting

$$
\begin{aligned}
v_{1}(x, y) & =v(x, y), \quad y \geqq 0 \\
& =\sum_{k=1}^{m_{n}} \lambda_{k} v(x,-k y), \quad y<0,
\end{aligned}
$$

where the $\lambda_{k}$ are constants chosen so that all the derivatives $D_{y}^{j} v$ for $0 \leqq j \leqq m_{n}-1$ 
are continuous at $y=0$. Here $\lambda_{k}$ depends only on $m_{n}$. We observe that for $\alpha$ satisfying $\langle\alpha, q\rangle \leqq m$

$$
\left[D^{\alpha} v_{1}\right]^{\sim}=\xi_{1}^{\alpha} \cdots \xi_{n-1}^{\alpha_{n}-1} \eta^{\alpha} \tilde{v}_{1}(\xi, \eta)
$$

and that, for any $\varepsilon>0$ and $\beta(\langle\beta, q\rangle<m)$,

$$
\left|\xi_{1}^{\beta_{1}} \cdots \xi_{n-1}^{\beta_{n}-1} \eta^{\beta_{n}}\right| \leqq \varepsilon \sum_{\langle\alpha, q\rangle=m}\left|\xi_{1}^{\alpha_{1}} \cdots \xi_{n-1}^{\alpha_{n}-1} \eta^{\alpha_{n}}\right|+C
$$

with a constant $C$ depending only on $\varepsilon$. By using Parseval's identity the lemma follows.

By Lemma 5.1 we have

$$
\begin{aligned}
\sum_{\langle\alpha, q\rangle \leqq m}\left\|D^{\alpha} v\right\| & =\sum_{\langle\alpha, q\rangle\langle m}\left\|D^{\alpha} v\right\|+\sum_{\langle\alpha, q\rangle=m}\left\|D^{\alpha} v\right\| \\
& \leqq(1+\varepsilon) \sum_{\langle\alpha, q\rangle=m}\left\|D^{\alpha} v\right\|+C(\varepsilon)\|v\|, \quad v \in C_{0}^{\infty}\left(\left(R_{+}^{n}\right)^{a}\right) .
\end{aligned}
$$

So, from Theorem 3.1 we get

$$
\begin{aligned}
\sum_{\langle\alpha, q\rangle \leqq m}\left\|D^{\alpha} v\right\| \leqq C\left(\left\|P^{0}(D) v\right\|+\sum_{j=1}^{r}\left|Q_{j}^{0}(D) v\right|_{m-p_{j}-\left(m / 2 m_{n}\right)}+\|v\|\right) & \\
v & \in C_{0}^{\infty}\left(\left(R_{+}^{n}\right)^{a}\right) .
\end{aligned}
$$

Similarly we can see that for any $\varepsilon>0$ there is a constant $C>0$ such that

$$
\left\|P^{0}(D) v\right\| \leqq \varepsilon \sum_{\langle\alpha, a\rangle=m}\left\|D^{\alpha} v\right\|+C\|v\|+\|P(D) v\|, \quad v \in C_{0}^{\infty}\left(\left(R_{+}^{n}\right)^{a}\right) .
$$

Taking $\varepsilon$ sufficiently small (for instance $\varepsilon=\frac{1}{2}$ ) we get the inequality

$$
\sum_{\langle\alpha, q\rangle \leq m}\left\|D^{\alpha} v\right\| \leqq C\left(\|P(D) v\|+\sum_{j=1}^{r}\left|Q_{j}^{0}(D) v\right|_{m-p_{j}-\left(m / 2 m_{n}\right)}+\|v\|\right) .
$$

It remains to replace $Q_{f}^{\circ}(D) v$ by $Q_{f}(D) v$ in (5.3). To do so, again we need to extend $v(x, y)$ to the whole space $R^{n}$ as in the proof of Lemma 5.1 and we denote the extension by $v_{1}(x, y)$.

For any $v \in C_{0}^{\infty}\left(\left(R_{+}^{n}\right)^{-}\right)$, we have by Schwarz's inequality

$$
\begin{aligned}
|\hat{v}(\xi, 0)|^{2} & \left.\leqq \frac{1}{2 \pi}\left(\int_{-\infty}^{\infty}\left|\hat{v}_{1}(\xi, \eta)\right| d \eta\right)\right)^{2} \\
& \leqq \frac{1}{2 \pi} \int_{-\infty}^{\infty}\left(1+\langle\xi\rangle^{2 m / m_{n}}+\eta^{2}\right)\left|v_{1}(\xi, \eta)\right|^{2} d \eta \int_{-\infty}^{\infty} \frac{d \eta}{1+\langle\xi\rangle^{2 m / m_{n}}+\eta^{2}}
\end{aligned}
$$

The last integral is equal to $\pi\left(1+\langle\xi\rangle^{2 m / m_{n}}\right)^{-1 / 2}$. Hence we get

$$
(1+\langle\xi\rangle)^{m-p_{j}-m / 2 m_{n}}\left|\xi^{\alpha^{\prime}} D_{y^{n}}^{\alpha_{n}} \hat{v}(\xi, 0)\right| \leqq C(1+\langle\xi\rangle)^{m-p_{j}}\left(\int\left|\xi^{\alpha^{\prime}} \eta^{\alpha_{n}} \hat{v}_{1}\right|^{2} d \eta\right)^{1 / 2}
$$

$$
+(1+\langle\xi\rangle)^{m-p_{j}-m / m_{n}}\left(\int\left|\xi^{\alpha^{\prime}} \eta^{\alpha_{n}+1} \hat{v}_{1}\right|^{2} d \eta\right)^{1 / 2} \text {. }
$$


Here we put $\alpha=\left(\alpha^{\prime}, \alpha_{n}\right), \alpha^{\prime}=\left(\alpha_{1}, \ldots, \alpha_{n-1}\right)$ and we assume $\langle\alpha, q\rangle=p_{j}\left(p_{j} \leqq m\right.$ $\left.-m / m_{n}\right)$. For the first term on the right hand side, the method used already gives

$$
(1+\langle\xi\rangle)^{m-p_{j}}\left|\xi^{\alpha^{\prime}} \eta^{\alpha_{n}}\right| \leqq C\left(1+\left|\xi_{1}\right|^{m_{1}}+\cdots+|\eta|^{m_{n}}\right), \quad(\xi, \eta) \in R^{n},
$$

for some constant $C$. Similarly for the second term, we have

$$
(1+\langle\xi\rangle)^{m-p_{j}-m / m_{n}}\left|\xi^{\alpha^{\prime}} \eta^{\alpha_{n}+1}\right| \leqq C\left(1+\left|\xi_{1}\right|^{m_{1}}+\cdots+|\eta|^{m_{n}}\right), \quad(\xi, \eta) \in R^{n} .
$$

Thus by extending $v$ to $v_{1}$ and by Parseval's formula, we get

$$
\left|D^{\alpha} v\right|_{m-p_{j}-m / 2 m_{n}} \leqq C \sum_{\langle\beta, q\rangle \leqq m}\left\|D^{\beta} v\right\|, \quad v \in C_{0}^{\infty}\left(\left(R_{+}^{n}\right)^{a}\right)
$$

for any $\alpha$ such that $\langle\alpha, q\rangle=p_{j}$.

By triangle inequality we have

$$
\left|Q_{j}^{0}(D) v\right|_{m-p_{j}-m / 2 m_{n}} \leqq\left|Q_{j}(D) v\right|_{m-p_{j}-m / 2 m_{n}}+C \sum_{\langle\alpha, q\rangle<p_{j}}\left|D^{\alpha} v\right|_{m-p_{j}-m / 2 m_{n}} .
$$

Hence a slight modification of the proof of Lemma 5.1 gives for any $\varepsilon>0$

$$
\begin{aligned}
\left|Q_{j}^{0}(D) v\right|_{m-p_{j}-m / 2 m_{n}} \leqq & \left|Q_{j}(D) v\right|_{m-p_{j}-m / 2 m_{n}}+\varepsilon \sum_{\langle\alpha, q\rangle=p_{j}}\left|D^{\alpha} v\right|_{m-p_{j}-m / 2 m_{n}} \\
& +C(\varepsilon)|v|_{m-p_{j}-m / 2 m_{n}}, \quad v \in C_{0}^{\infty}\left(\left(R_{+}^{n}\right)^{a}\right) .
\end{aligned}
$$

By the argument used in the proof of the inequality (5.5) we get for the third term on the right hand side of (5.6)

$$
|v|_{m-p_{j}-m / 2 m_{n}} \leqq \varepsilon \sum_{\langle\alpha, q\rangle=m}\left\|D^{\alpha} v\right\|+C(\varepsilon)\|v\|, \quad v \in C_{0}^{\infty}\left(\left(R_{+}^{n}\right)^{a}\right) .
$$

Here we may assume $p_{j}>0$, otherwise we do not need such an inequality because $p_{j}=0$ implies $Q_{j}=$ const. and $Q_{j}^{0}=Q_{j}$.

Combining the inequalities (5.5), (5.6), and (5.7) we have

$$
\left|Q_{j}^{0}(D) v\right|_{m-p_{j}-m / 2 m_{n}} \leqq\left|Q_{j}(D) v\right|_{m-p_{j}-m / 2 m_{n}}+2 \varepsilon \sum_{\langle\alpha, q\rangle \leqq m}\left\|D^{\alpha} v\right\|+C\|v\| .
$$

Finally, taking $\varepsilon$ sufficiently small we arrive at the conclusion:

$$
\sum_{\langle\alpha, q\rangle \leqq m}\left\|D^{\alpha} v\right\| \leqq C\left(\|P(D) v\|+\sum_{j=1}^{r}\left|Q_{j}(D) v\right|_{m-p_{j}-m / 2 m_{n}}+\|v\|\right),
$$

$$
v \in C_{0}^{\infty}\left(\left(R_{+}^{n}\right)^{a}\right)
$$

This completes the proof of Theorem 3.2.

6. Coerciveness inequality (II). The case of variable coefficients. The conclusions of Theorem 3.1 and Theorem 3.2 can be extended to operators with variable coefficients.

Let $\Omega$ be a domain in $R_{+}^{n}$. It is supposed that the boundary of $\Omega$ contains an open set $\omega(\neq \varnothing)$ in the plane $y=0$. For convenience, assume the origin $(0, \ldots, 0)$ is contained in the (interior of) plane boundary $\omega$. 
We consider a linear differential operator

$$
P(x, y, D)=\sum_{\langle\alpha, q\rangle \leqq m} a_{\alpha}(x, y) D^{\alpha},
$$

where $a_{\alpha}(x, y)$ are complex valued functions defined on $\Omega \cup \omega$ and infinitely differentiable. We assume that the operator (6.1) is quasi-elliptic of weight $q=\left(m / m_{1}, \ldots, m / m_{n}\right)$ in $\Omega \cup \omega$, more precisely, there is a constant $K>0$ such that

$$
\left|\xi_{1}\right|^{m_{1}}+\cdots+\left|\xi_{n-1}\right|^{m_{n-1}}+|\eta|^{m_{n}} \leqq K\left|\sum_{\langle\alpha, a\rangle=m} a_{\alpha}(x, y) \xi^{\alpha^{\prime}} \eta^{\alpha_{n}}\right|
$$

for any $(\xi, \eta) \in R^{n}$ and for any $(x, y) \in \Omega \cup \omega$. We may assume the coefficient of $D_{n}^{m_{n}}$ is identically equal to 1 .

Set $P(D)=\sum_{\langle\alpha, q\rangle \leqq m} a_{\alpha}(0,0) D^{\alpha}$ and assume that $P(D)$ is of determined type $r$, $1 \leqq r \leqq m_{n}$ (see $\S 1$ ). We consider $r$ boundary operators $Q_{j}(x, D)$ defined on $\omega$ :

$$
Q_{j}(x, D)=\sum_{\langle\alpha, q\rangle \leqq p_{j}} b_{\alpha}(x) D_{x}^{\alpha^{\prime}} D_{y^{n}}^{\alpha_{n}}, \quad j=1, \ldots, r, 0 \leqq p_{j} \leqq m-m / m_{n},
$$

where $b_{\alpha}(x)$ are complex valued functions defined on $\omega$ and infinitely differentiable. Set

$$
Q_{j}(D)=\sum_{\langle\alpha, q\rangle \leqq p_{j}} b_{\alpha}(0) D_{x}^{\alpha^{\prime}} D_{y^{n}}^{\alpha_{n}}, \quad j=1, \ldots, r .
$$

We denote by $\Omega_{\delta}(\delta>0)$ the hemisphere

$$
\Omega_{\delta}=\left\{(x, y) ; y \geqq 0,|x|^{2}+y^{2}<\delta^{2}\right\},
$$

and denote by $\omega_{\delta}$ the plane boundary of $\Omega_{\delta}$ :

$$
\omega_{\delta}=\{(x, 0) ; x<\delta\} .
$$

TheOREM 6.1. Let $P(x, y, D)$ and $Q_{j}(x, D), j=1, \ldots, r$ be defined as above and assume that $Q_{j}(D)(j=1, \ldots, r)$ cover $P(D)$. Then for sufficiently small $\delta>0$ there exists a constant $C>0$ such that

$$
\begin{aligned}
\sum_{\langle\alpha, q\rangle \leq m}\left\|D^{\alpha} v\right\| \leqq C\left(\|P(x, y, D) v\|+\sum_{j=1}^{r}\left|Q_{j}(x, D) v\right|_{m-p_{j}-m / 2 m_{n}}+\|v\|\right) & \\
v & \in C_{0}^{\infty}\left(\Omega_{\delta}\right) .
\end{aligned}
$$

7. Proof of Theorem 6.1. In view of Theorem 3.2 it holds for some constant $C$

$$
\sum_{\langle\alpha, a\rangle \leq m}\left\|D^{\alpha} v\right\| \leqq C\left(\|P(D) v\|+\sum_{j=1}^{r}\left|Q_{j}(D) v\right|_{m-p_{j}-m / 2 m_{n}}+\|v\|\right),
$$

$$
v \in C_{0}^{\infty}\left(\left(R_{+}^{n}\right)^{a}\right) \text {. }
$$

Let us write

$$
P(D) v=P(x, y, D) v+[P(D)-P(x, y, D)] v .
$$


Then for any $\varepsilon>0$ we can take sufficiently small $\delta>0$ such that

$$
\|P(D) v\| \leqq\|P(x, y, D) v\|+\varepsilon \sum_{\langle\alpha, q\rangle \leqq m}\left\|D^{\alpha} v\right\|, \quad v \in C_{0}^{\infty}\left(\Omega_{\delta}\right) .
$$

Put $\varepsilon=1 / 2$. Then we have

$$
\sum_{\langle\alpha, q\rangle \leqq m}\left\|D^{\alpha} v\right\| \leqq 2 C\left(\|P(x, y, D) v\|+\sum_{j=1}^{r}\left|Q_{j}(D) v\right|_{m-p_{j}-m / 2 m_{n}}+\|v\|\right),
$$

It remains to replace $Q_{j}(D)$ by $Q_{j}(x, D)$ in (7.3). To do so, we shall prove some lemmas. We denote by $\gamma_{s} \in \mathbb{S}^{\prime}\left(R^{n-1}\right)$ the distribution such that

$$
\hat{\gamma}_{s}(\xi)=\left(1+\xi_{1}^{2 m_{1}}+\cdots+\xi_{n-1}^{2 m_{n}-1}\right)^{s / 2 m}
$$

for real $s$.

Lemma 7.1 (CF. Mizohata [9]). Let s be real and positive. Then

$$
\left\|\left(x^{\alpha} \gamma_{s}\right) * \varphi\right\| \leqq \varepsilon(\alpha, \delta)\left\|\gamma_{s} * \varphi\right\|, \quad \varphi \in C_{0}^{\infty}\left(R^{n-1}\right), \quad \alpha \neq 0 .
$$

Here, $\varepsilon(\alpha, \delta)$ is a constant such that $\varepsilon(\alpha, \delta)$ tends to zero when the diameter $\delta$ of the support of $\varphi$ tends to zero.

Proof. It is obvious that

$$
x^{\alpha} \gamma_{s}(\xi)=(-1)^{|\alpha|} D_{\xi}^{\alpha} \hat{\gamma}_{s}(\xi) \equiv P_{\alpha}(\xi) \hat{\gamma}_{s}(\xi)
$$

where $P_{\alpha}(\xi)$ tends to zero when $|\xi| \rightarrow \infty$. Now

$$
\left(x^{\alpha} \gamma_{s}\right) * \varphi(x)=(2 \pi)^{-(n-1) / 2} \int_{|\xi| \leqq R} \exp (i\langle x, \xi\rangle) P_{\alpha}(\xi) \hat{\gamma}_{s}(\xi) \hat{\varphi}(\xi) d \xi+\int_{|\xi| \geqq R} \cdots
$$

Take $R$ sufficiently large. Then $\left|P_{\alpha}(\xi)\right| \leqq \varepsilon / 2,|\xi|>R$. The $L^{2}$-norm of the second term is dominated by $\varepsilon / 2\left\|\gamma_{s} * \varphi\right\|$, where $\varepsilon$ will be given later. Let $R$ be fixed as above. The $L^{2}$-norm of the first term is estimated by

$$
\max _{|\xi| \leqq R}\left|P_{\alpha}(\xi) \hat{\gamma}_{s}(\xi)\right|\left(\int_{|\xi| \leqq R}|\hat{\varphi}(\xi)|^{2} d \xi\right)^{1 / 2} .
$$

On the other hand, it holds that

$$
\left.|\hat{\varphi}(\xi)| \leqq \int|\varphi(x)| d x \leqq(\text { Vol. [supp. } \varphi]\right)^{1 / 2} \cdot\|\varphi\| .
$$

Hence (7.6) is estimated by

$$
\left.\max \left|P_{\alpha}(\xi) \hat{\gamma}_{s}(\xi)\right|(\text { Vol. [supp. } \varphi]\right)^{1 / 2} \cdot\|\varphi\| \cdot(\text { Vol. } B(R))^{1 / 2},
$$

where $B(R)$ is a ball whose radius is $R$. Clearly $\|\varphi\| \leqq\left\|\gamma_{s} * \varphi\right\|$ for $s>0$. So the lemma is proved. 
REMARK. We note that $x^{\alpha} \gamma_{s}(x) \in L^{2}\left(R^{n-1}\right)$ if we take $\alpha$ such that $|\alpha|$ is sufficiently large.

LEMMA 7.2 (CF. [9]). Let $s$ be real, positive and $b(x) \in C^{\infty}\left(R^{n-1}\right)$. Then

$$
\left\|b(x)\left(\gamma_{s} * \varphi\right)-\gamma_{s} *(b(x) \varphi)\right\| \leqq \varepsilon(\delta, s)\left\|\gamma_{s} * \varphi\right\|, \quad \varphi \in C_{0}^{\infty}\left(R^{n-1}\right) .
$$

Here $\varepsilon(\delta, s)$ is a constant, which tends to zero when the diameter of the support of $\varphi$ tends to zero.

Proof. The commutator, which we must estimate, is

$$
b(x) \gamma_{s} * \varphi-\gamma_{s} * b(x) \varphi=\int\left[b(x)-b\left(x^{\prime}\right)\right] \gamma_{s}\left(x-x^{\prime}\right) \varphi\left(x^{\prime}\right) d x^{\prime} .
$$

We use the Taylor's formula for $b\left(x^{\prime}\right)$ around $x$ :

$$
b\left(x^{\prime}\right)-b(x)=\sum_{1 \leqq|\alpha| \leqq l-1} \frac{\left(x^{\prime}-x\right)^{\alpha}}{\alpha !} D^{\alpha} b(x)+\sum_{|\alpha|=l} b_{\alpha}\left(x, x^{\prime}\right)\left(x^{\prime}-x\right)^{\alpha}, \quad b_{\alpha}\left(x, x^{\prime}\right) \in C^{\infty} .
$$

Then (7.8) is written as

$$
\begin{aligned}
& \sum_{1 \leqq|\alpha| \leqq l-1}(-1)^{\alpha} \frac{D^{\alpha} b(x)}{\alpha !}\left(x^{\alpha} \gamma_{s}\right) * \varphi \\
& +(-1)^{l+1} \sum_{|\alpha|=l} \int b_{\alpha}\left(x, x^{\prime}\right)\left(x-x^{\prime}\right)^{\alpha} \gamma_{s}\left(x-x^{\prime}\right) \varphi\left(x^{\prime}\right) d x^{\prime}
\end{aligned}
$$

By Lemma 7.1 it is clear that every expression $\left(D^{\alpha} b(x) / \alpha !\right)\left(x^{\alpha} \gamma_{s}\right) * \varphi$ in the first term of (7.9) has the property (7.7). We take $l$ sufficiently large so that $x^{\alpha} \gamma_{s}(x) \in L^{2}$, $|\alpha|=l$ (cf. Remark of Lemma 7.1). Then every term in the second summation is estimated as follows:

$$
\sup \left|b_{\alpha}\left(x, x^{\prime}\right)\right| \int\left|\left(x-x^{\prime}\right)^{\alpha} \gamma_{s}\left(x-x^{\prime}\right)\right|\left|\varphi\left(x^{\prime}\right)\right| d x^{\prime}=\sup \left|b_{\alpha}\left(x, x^{\prime}\right)\right|\left(\left|x^{\alpha} \gamma_{s}\right| *|\varphi|\right) .\left(^{2}\right)
$$

By Hausdorff-Young's inequality

$$
\left\|\left|x^{\alpha} \gamma_{s}\right| *|\varphi|\right\|_{L^{2}} \leqq\left\|x^{\alpha} \gamma_{s}\right\|_{L^{2}} \cdot\|\varphi(x)\|_{L^{1}}
$$

Moreover

$$
\left.\|\varphi(x)\|_{L^{1}} \leqq(\text { Vol. [supp. } \varphi]\right)^{1 / 2} \cdot\|\varphi(x)\|_{L^{2}} .
$$

Combining these estimates we obtain the inequality (7.7)

CoRollary 1. Let $\varphi \in C_{0}^{\infty}\left(R^{n-1}\right)$, supp. $\varphi \subset\{x ;|x| \leqq \delta\} \equiv B(\delta)$ and $b(x) \in C^{\infty}\left(R^{n-1}\right)$ with $b$ and each $D^{\alpha} b$ bounded on $R^{n-1}$. Then

$$
\left\|\gamma_{s} * b(x) \varphi\right\| \leqq C|b|_{k}\left\|\gamma_{s} * \varphi\right\|, \quad k=k(s),-\infty<s<\infty
$$

(2) It follows easily from our hypotheses that $b_{\alpha}\left(x, x^{\prime}\right)$ is bounded on $R^{n-1} \times R^{n-1}$. 
where

$$
|b|_{k}=\sum_{|\alpha| \leqq k} \sup _{x \in R^{n-1}}\left|D^{\alpha} b(x)\right| .
$$

In particular, if $b(0)=0$, then

$$
\left\|\gamma_{s} * b(x) \varphi\right\| \leqq \varepsilon(\delta, s)\left\|\gamma_{s} * \varphi\right\|,
$$

where $\varepsilon(\delta, s)$ is a constant such that $\varepsilon(\delta, s) \rightarrow 0$ when the diameter $\delta$ of the support of $\varphi$ tends to zero.

The proof is easy, so we omit it here.

Now let us continue the proof of Theorem 6.1. We note that the norms $|v|_{s}$ and $\left\|\gamma_{s} * v(x, 0)\right\|_{L^{2}\left(R^{n-1}\right)}$ are equivalent to each other. Let us write

$$
Q_{j}(D) v(x, 0)=Q_{j}(x, D) v+\left(Q_{j}(D)-Q_{j}(x, D)\right) v, \quad v \in C_{0}^{\infty}\left(\Omega_{\delta}\right) .
$$

If we assume that $\delta<1$, we may (by altering the coefficients of the $b_{j}(x, D)$ outside the unit ball) assume that coefficients of $b_{j}(x, D)$ and each of its derivatives is bounded on $R^{n-1}$. Then the corollary stated above yields

$$
\begin{aligned}
\left|Q_{j}(D) v\right|_{m-p_{j}-m / 2 m_{n}} \leqq & \left|Q_{j}(x, D) v\right|_{m-p_{j}-m / 2 m_{n}} \\
& +\varepsilon\left(\delta, p_{j}\right) \sum_{\langle\alpha, q\rangle \leqq p_{j}}\left|D^{\alpha} v\right|_{m-p_{j}-m / 2 m_{n}}, \quad v \in C_{0}^{\infty}\left(\Omega_{\delta}\right) .
\end{aligned}
$$

As we have seen in the proof of Theorem 3.2 there exists a constant $C$ not depending on $\delta$ such that

$$
\sum_{\langle\alpha, q\rangle \leqq p_{j}}\left|D^{\alpha} v\right|_{m-p_{j}-m / 2 m_{n}} \leqq C \sum_{\langle\alpha, q\rangle \leqq m}\left\|D^{\alpha} v\right\|, \quad v \in C_{0}^{\infty}\left(\Omega_{\delta}\right) .
$$

Hence if we take $\delta$ sufficiently small, we get the estimate:

$\sum_{\langle\alpha, q\rangle \leqq m}\left\|D^{\alpha} v\right\| \leqq C\left(\|P(x, y, D) v\|+\sum_{j=1}^{r}\left|Q_{j}(x, D) v\right|_{m-p_{j}-m / 2 m_{n}}+\|v\|\right), \quad v \in C_{0}^{\infty}\left(\Omega_{\delta}\right)$, which completes the proof.

REMARK. We note that the necessity of our complementing condition (Definition 3.1) for the validity of the estimate (3.8) can be proved by the analogous way in $[1, \S 10]$.

8. Regularity at the boundary. We introduce function spaces $\Phi^{m}$ and $H^{s}$.

Definition 8.1. By $\Phi^{m}\left(R_{+}^{n}\right)=\Phi^{m}$ we mean the set of all $u \in L^{2}\left(R_{+}^{n}\right)$ such that $D^{\alpha} u \in L^{2}\left(R_{+}^{n}\right)$ for any $\alpha$ satisfying $\langle\alpha, q\rangle \leqq m$. The space $\Phi^{m}$ is a Hilbert space with norm

$$
\|u\|_{m}^{2}=\sum_{\langle\alpha, q\rangle \leqq m}\left\|D^{\alpha} u\right\|^{2} .
$$

We denote by $H^{s}\left(R^{n-1}\right)=H^{s}$ (for a real $s \geqq 0$ ) the set of all $u \in L^{2}\left(R^{n-1}\right)$ such that

$$
(1+\langle\xi\rangle)^{s} \hat{u}(\xi) \in L^{2}\left(R^{n-1}\right) .
$$


The space $H^{s}$ is a Hilbert space with norm

$$
|u|_{s}=\left\|(1+\langle\xi\rangle)^{s} \hat{u}(\xi)\right\|_{L^{2}\left(R^{n-1}\right)} \cong\left\|\gamma_{s} * u\right\|_{L^{2}\left(R^{n-1}\right)},
$$

where we also denote by $\hat{u}(\xi)$ the Fourier transform of $u$ with respect to variables $x=\left(x_{1}, \ldots, x_{n-1}\right)$.

Definition 8.2. We shall say $u \in \Phi_{\mathrm{loc}}^{m}\left(\Omega_{\delta}\right)$ if $\varphi u \in \Phi^{m}$ for all $\varphi \in C_{0}^{\infty}\left(\Omega_{\delta}\right)$. Similarly we shall say $u \in H_{\mathrm{loc}}^{s}\left(\omega_{\delta}\right)$ if $\varphi u(x) \in H^{s}$ for all $\varphi \in C_{0}^{\infty}\left(\omega_{\delta}\right)$.

Concerning with the properties of the spaces $\Phi^{m}$ and $H^{s}$ we have the following lemmas.

LemMa 8.1. Let $s$ and $t$ be two integers such that $0<s<t$. Then for any $\varepsilon>0$ there exists a constant $C=C(\varepsilon)$ such that

$$
\|u\|_{s} \leqq \varepsilon\|u\|_{t}+C\|u\|, \quad u \in \Phi^{t}
$$

Proof. Clearly $C_{0}^{\infty}\left(\left(R_{+}^{n}\right)^{a}\right)$ is dense in $\Phi^{t}$ for every $t$. Hence Lemma 8.1 can be reduced to Lemma 5.1 .

LEMma 8.2. For every $u \in \Phi^{m}$ there exists the trace

$$
D^{\alpha} u(x, 0) \in H^{m-p-m / 2 m_{n}}, \quad 0 \leqq p \leqq m-m / m_{n},\langle\alpha, q\rangle \leqq p,
$$

and there is a constant $C=C(m)$ such that

$$
\sum_{\langle\alpha, q\rangle \leqq p}\left|D^{\alpha} u\right|_{m-p-m / 2 m_{n}} \leqq C\|u\|_{m}, \quad u \in \Phi^{m}
$$

The proof of Lemma 8.2 can be given by the argument stated at the end of $\S 5$. So we omit the detail.

Now take $\rho=\rho(x) \in C_{0}^{\infty}\left(R^{n-1}\right)$ satisfying the following:

(i) $\int_{R^{n-1}} \rho(x) d x=1$ and

(ii) $\rho(\xi)=O\left(|\xi|^{k}\right), \xi \rightarrow 0$ for some sufficiently large integer $k$.

Set $\rho_{\varepsilon}(x)=\varepsilon^{-(n-1)} \rho(x / \varepsilon)(\varepsilon>0)$. If $v \in \Phi^{m}$, the regularization of $v$

$$
v_{\varepsilon}(x, y)=\varepsilon^{-(n-1)} \int v\left(x^{\prime}, y\right) \rho\left(x-x^{\prime} / \varepsilon\right) d x^{\prime}, \quad y \geqq 0,
$$

is infinitely differentiable with respect to $x$ and obviously $v_{\varepsilon} \in \Phi^{m}$, and $v_{\varepsilon} \rightarrow v$ in $\Phi^{m}\left(R_{+}^{n}\right)$ as $\varepsilon \rightarrow 0$.

As usual we denote by $\mathfrak{S}\left(\left(R_{+}^{n}\right)^{a}\right)$ the set of all the functions, each of which is a restriction in $\left(R_{+}^{n}\right)^{a}$ of a function in $S\left(R^{n}\right)$.

LEMMA 8.3. If $u \in L^{2}\left(R_{+}^{n}\right)$ and $a \in \mathfrak{S}\left(\left(R_{+}^{n}\right)^{a}\right)$ and if $\rho \in C_{0}^{\infty}\left(R^{n-1}\right)$ satisfies (i) and (ii), then

$$
\left\|\gamma_{1} *\left(a u_{\varepsilon}-(a u)_{\varepsilon}\right)\right\|_{L^{2}\left(R_{+}^{n}\right)} \rightarrow 0 \quad \text { as } \quad \varepsilon \rightarrow 0 .
$$

The lemma is derived by a simple modification of the proof of Theorem 2.4.3 in [5], so we omit the proof. (For the definition of $\gamma_{1}$, see (7.4).) 
THEOREM 8.1. Consider the boundary problem

$$
\begin{aligned}
P(x, y, D) u=f & \text { in } \Omega, \\
Q_{j}(x, D) u=g_{j} & \text { on } \quad \omega, \quad j=1, \ldots, r,
\end{aligned}
$$

where $P(x, y, D)$ is a quasi-elliptic operator given in $\$ 6$, and the boundary operators $Q_{j}(x, D)$ are also given in $\$ 6$.

Suppose $u \in \Phi_{\mathrm{loc}}^{m}(\Omega)$. If $f \in C^{\infty}(\bar{\Omega})$ and if $g_{j} \in C^{\infty}(\omega), j=1, \ldots, r$, then there exists a positive number $\delta=\delta(t)$ such that $u \in \Phi_{10 \mathrm{c}}^{m+t}\left(\Omega_{\delta}\right)$ for $t=1,2, \ldots$

Proof. First take any tangential derivative $D_{x}^{\beta}$ (here $\beta=\left(\beta_{1}, \ldots, \beta_{n-1}, 0\right)$ ). We want to prove that there exists a $\delta=\delta(\beta)$ such that

$$
D_{x}^{\beta}(\varphi u) \in \Phi^{m}
$$

for any $\varphi \in C_{0}^{\infty}\left(\Omega_{\delta}\right)$.

To do so, it is sufficient to prove that for any positive integer $s$ there exists a $\delta=\delta(s)$ such that

$$
\gamma_{s} *(\varphi u) \in \Phi^{m}
$$

for any $\varphi \in C_{0}^{\infty}\left(\Omega_{\delta}\right)$. We consider the case $s=1$.

We set $P(D)=\sum_{\langle\alpha, q\rangle \leqq m} a_{\alpha}(0,0) D^{\alpha}$ and $Q_{f}(D)=\sum_{\langle\alpha, q\rangle \leqq p_{j}} b_{\alpha}(0) D^{\alpha}, j=1, \ldots, r$, the same as in $\S 6$. As $\gamma_{1} *(\varphi u)_{\varepsilon} \in \Phi^{m}\left(R_{+}^{n}\right)$ for any $\varphi \in C_{0}^{\infty}(\Omega)$ and for any $\varepsilon>0$, the coerciveness estimate yields

$$
\begin{aligned}
\left\|\gamma_{1} *(\varphi u)_{\varepsilon}\right\|_{m} \leqq & C\left(\left\|P(D)\left(\gamma_{1} *(\varphi u)_{\varepsilon}\right)\right\|\right. \\
& \left.+\sum_{j=1}^{r}\left|Q_{j}(D) \gamma_{1} *(\varphi u)_{\varepsilon}\right|_{m-p_{j}-m / 2 m_{n}}+\left\|\gamma_{1} *(\varphi u)_{\varepsilon}\right\|\right),
\end{aligned}
$$

where the constant $C$ is independent of $\varphi \in C_{0}^{\infty}(\Omega)$ and of $\varepsilon(>0)$.

Write

$$
\begin{aligned}
\gamma_{1} * P(D)(\varphi u)_{\varepsilon}= & \gamma_{1} * P(x, y, D)(\varphi u)_{\varepsilon} \\
& +\gamma_{1} *\left\{(P(D)-P(x, y, D))(\varphi u)_{\varepsilon}\right\}
\end{aligned}
$$

Then, for the second term in the right hand side, we can easily see by using Corollary 1 in $\S 7$, that for any $\nu>0$ there exist $\delta=\delta(\nu)$ and $\varepsilon_{0}=\varepsilon_{0}(\nu)$ such that

$$
\left\|\gamma_{1} *(P(D)-P(x, y, D))(\varphi u)_{\varepsilon}\right\|_{L^{2}\left(R_{+}^{n}\right)} \leqq \nu\left\|\gamma_{1} *(\varphi u)_{\varepsilon}\right\|_{m}
$$

for any $\varphi \in C_{0}^{\infty}\left(\Omega_{\delta}\right)$ and for any $\varepsilon\left(0<\varepsilon \leqq \varepsilon_{0}\right)$. Similarly we can get

$$
\left|\gamma_{1} *\left\{Q_{j}(D)-Q_{j}(x, D)(\varphi u)_{\varepsilon}\right\}\right|_{m-p_{j}-m / 2 m_{n}} \leqq \nu\left\|\gamma_{1} *(\varphi u)_{\varepsilon}\right\|_{m},
$$

$j=1, \ldots, r$, for any $\varphi \in C_{0}^{\infty}\left(\Omega_{\delta}\right)$ and for any $\varepsilon\left(0<\varepsilon \leqq \varepsilon_{0}\right)$. Here also $\delta$ and $\varepsilon_{0}$ depend only on $\nu$. 
Thus we get the following estimate:

$$
\begin{aligned}
\left\|\gamma_{1} *(\varphi u)_{\varepsilon}\right\|_{m} \leqq & C\left(\left\|\gamma_{1} * P(x, y, D)(\varphi u)_{\varepsilon}\right\|\right. \\
& +\sum_{j=1}^{r}\left|\gamma_{1} * Q_{j}(x, D)(\varphi u)_{\varepsilon}\right|_{m-p_{j}-m / 2 m_{n}} \\
& \left.+\left\|\gamma_{1} *(\varphi u)_{\varepsilon}\right\|\right), \quad \varphi \in C_{0}^{\infty}\left(\Omega_{\delta}\right), \quad 0<\varepsilon \leqq \varepsilon_{0},
\end{aligned}
$$

where the constant $C$ is independent of $\varphi$ and $\varepsilon$.

Next we prove that the norms

and

$$
\left\|\gamma_{1} * P(x, y, D)(\varphi u)_{\varepsilon}\right\|
$$

$$
\left|\gamma_{1} * Q_{j}(x, D)(\varphi u)_{6}\right|_{m-p,-m / 2 m_{n}}, \quad j=1, \ldots, r,
$$

are uniformly bounded with respect to $\varepsilon\left(0<\varepsilon \leqq \varepsilon_{0}\right)$. By Lemma 8.3 it is sufficient to prove the uniform boundedness of the norms

$$
\| \gamma_{1} *\left(P(x, y, D)(\varphi u)_{\varepsilon} \|\right.
$$

and

$$
\left|\gamma_{1} *\left(Q_{j}(x, D)(\varphi u)_{\varepsilon}\right)\right|_{m-p_{j}-m / 2 m_{n}}, \quad j=1, \ldots, r .
$$

We note that $\gamma_{1} *(P(x, y, D)(\varphi u))_{\varepsilon}=\left(\gamma_{1} * P(x, y, D)(\varphi u)\right)_{\varepsilon}$. If we write

$$
P(x, y, D)(\varphi u)=\varphi P(x, y, D) u+\sum_{\beta \neq 0} \frac{D^{\beta} \varphi}{\beta !} \cdot P^{\beta}(x, y, D) u,
$$

then the fact $\varphi P(x, y, D) u=\varphi f \in C_{0}^{\infty}\left(\Omega_{\delta}\right)$ implies $\gamma_{1} * \varphi f \in L^{2}\left(R_{+}^{n}\right)$. Now, we take any term $\left(D^{\beta} \varphi / \beta !\right) a_{\alpha}(x, y) D^{\alpha-\beta} u$ in the summation of (8.12). Take $\psi \in C_{0}^{\infty}\left(\Omega_{\delta}\right)$ such that $\psi=1$ in supp. $\varphi$. Then

$$
\left(D^{\beta} \varphi / \beta !\right) a_{\alpha}(x, y) D^{\alpha-\beta} u=\left(D^{\beta} \varphi / \beta !\right) a_{\alpha}(x, y) D^{\alpha-\beta}(\psi u) .
$$

By virtue of Lemma 7.2 it is sufficient to prove $\gamma_{1} * D^{\alpha-\beta}(\psi u) \in L^{2}\left(R_{+}^{n}\right)$. We can easily observe that if $\langle\alpha, q\rangle \leqq m, \beta \leqq \alpha, \beta \neq 0$, then there exists a constant $C$ such that

$$
\begin{aligned}
\left(1+\left|\xi_{1}\right|^{m_{1} / m}+\cdots+\right. & \left.+\left|\xi_{n-1}\right|^{m_{n-1} / m}\right)\left|\xi^{\alpha^{\prime}-\beta^{\prime}} \eta^{\alpha_{n}-\beta_{n}}\right| \\
& \leqq C\left(1+\left|\xi_{1}\right|^{m_{1}}+\cdots+\left|\xi_{n-1}\right|^{m_{n-1}}+|\eta|^{m_{n}}\right)
\end{aligned}
$$

for any $(\xi, \eta) \in R^{n}$. Let $(\psi u)_{1}$ be the extension of $\psi u$ in $\Phi^{m}\left(R^{n}\right)$ by the method used in the proof of Lemma 5.1. Then $\gamma_{1} * D^{\alpha-\beta}(\psi u)_{1} \in L^{2}\left(R^{n}\right)$. Therefore we see $\gamma_{1} * D^{\alpha-\beta}(\psi u) \in L^{2}\left(R_{+}^{n}\right)$. Hence we have

$$
\gamma_{1} * P(x, y, D)(\varphi u) \in L^{2}\left(R_{+}^{n}\right) .
$$

Now it is obvious that

$$
\left(\gamma_{1} * P(x, y, D)(\varphi u)\right)_{\varepsilon}=\gamma_{1} *(P(x, y, D)(\varphi u))_{\varepsilon}
$$

is uniformly bounded for $\varepsilon\left(0<\varepsilon \leqq \varepsilon_{0}\right)$. 
For the norms $\left|\gamma_{1} * Q_{j}(x, D)(\varphi u)\right|_{m-p_{j}-m / 2 m_{n}}$, we again write

$$
Q_{j}(x, D)(\varphi u)=\varphi Q_{j}(x, D) u+\sum_{\beta \neq 0} \frac{D^{\beta} \varphi}{\beta !} Q_{j}^{\beta}(x, D) u .
$$

Similarly to the above, it is sufficient to prove that

$$
\left|\gamma_{1} * D^{\alpha-\beta}(\psi u)\right|_{m-p_{j}-m / 2 m_{n}}<\infty
$$

for any $\psi \in C_{0}^{\infty}\left(\Omega_{\delta}\right)$, where $\langle\alpha, q\rangle \leqq p_{j}, \beta \leqq \alpha, \beta \neq 0$. Clearly, it holds that

$$
\left|\gamma_{1} * D^{\alpha-\beta}(\psi u)\right|_{m-p_{j}-m / 2 m_{n}} \leqq\left|D^{\alpha-\beta}(\psi u)\right|_{m-\left(p_{j}-1\right)-m / 2 m_{n}},
$$

and $\langle\alpha-\beta, q\rangle \leqq p_{j}-1$. So Lemma 8.2 gives us

$$
\left|\gamma_{1} * D^{\alpha-\beta}(\psi u)\right|_{m-p_{j}-m / 2 m_{n}}<\infty .
$$

Thus, by using Lemma 8.3 , we can conclude that the norms

$$
\left\|\gamma_{1} *\left(P(x, y, D)(\varphi u)_{\varepsilon}\right)\right\|
$$

and

$$
\left|\gamma_{1} * Q_{j}(x, D)(\varphi u)_{\varepsilon}\right|_{m-p_{j}-m / 2 m_{n}}, \quad j=1, \ldots, r,
$$

are uniformly bounded with respect to $\varepsilon\left(0<\varepsilon \leqq \varepsilon_{0}\right)$. By the estimate (8.11), we can see that the norm $\left\|\gamma_{1} *(\varphi u)\right\|_{m}$ is uniformly bounded with respect to $\varepsilon\left(0<\varepsilon \leqq \varepsilon_{0}\right)$. Hence the theorem of Banach-Saks implies $\gamma_{1} * \varphi u \in \Phi^{m}$.

We can repeat this procedure and get

$$
D_{x}^{\beta} u \in \Phi_{\mathrm{loc}}^{m}\left(\Omega_{\delta}\right), \quad|\beta| \leqq N, \quad \beta_{n}=0, \quad \delta=\delta(N)
$$

for any integer $N$. Now the equation $P(x, y, D) u=f$ can be written in the form

$$
D_{y^{n}}^{m_{n}} u=-\sum_{\langle\alpha, q\rangle \leqq m ; \alpha_{n}<m_{n}} a_{\alpha}(x, y) D_{x}^{\alpha^{\prime}} D_{y^{n}}^{\alpha} u+f .
$$

Differentiating (8.13) with respect to $x$-variables we see

$$
D_{x}^{\beta} D_{y}^{m_{n}} u \in L_{\mathrm{loc}}^{2}\left(\Omega_{\delta}\right) \text {. }
$$

Moreover we have

$$
D_{x}^{\beta} D_{y}^{m_{n}+1} u \in L_{\text {loc }}^{2}\left(\Omega_{\delta}\right) .
$$

We can repeat this procedure and arrive at the conclusion of Theorem 8.1.

9. Hypo-analyticity at the boundary. In the following we shall investigate the more precise estimates of the derivatives of the solutions of a quasi-elliptic boundary problem. However, we are limited to the case of simple boundary operators.

Consider a quasi-elliptic operator $P(D)$ of weight $q=\left(m / m_{1}, \ldots, m / m_{n-1}, m / m_{n}\right)$ and of determined type $r\left(1 \leqq r \leqq m_{n}\right)$ given by (3.1):

$$
P(D)=D_{y}^{m_{n}}+\sum_{\langle\alpha, q\rangle \leqq m ; \alpha_{n}<m_{n}} a_{\alpha} D_{x}^{\alpha^{\prime}} D_{y^{n}}^{\alpha_{n}}
$$

where $a_{\alpha}$ are complex constants. 
As the boundary operators we take only normal derivatives such that $Q_{j}(D)=D_{y^{\prime}}^{k_{j}}, j=1, \ldots, r$, where $k_{i} \neq k_{j}$ if $i \neq j$ and $0 \leqq k_{j} \leqq m_{n}-1$. Clearly these $r$ boundary operators cover $P(D)$ (see Definition 3.1).

Definition 9.1. Let $\Omega$ be a domain in $R^{n}$ and set $d=\left(d_{1}, \ldots, d_{n}\right), d_{i} \geqq 1, i=$ $1, \ldots, n$. We call $u$ a function of the class $G(d, \Omega)$ if $u$ is infinitely differentiable in $\Omega$ and if for each compact set $K$ in $\Omega$ there exist two constants $C_{0}, C_{1}$ such that

$$
\left\|D^{\alpha} u, K\right\|_{\infty} \leqq C_{0} C_{1}^{|\alpha|} \prod_{i=1}^{n} \alpha_{i}^{d_{i} \alpha_{i}}
$$

or equivalently (if some $\alpha_{i}=0$, then we define $\alpha_{i}^{\alpha_{i}}=1$ and $|\alpha|^{|\alpha|}=1$ if $|\alpha|=0$ )

$$
\left\|D^{\alpha} u, K\right\|_{\infty} \leqq C_{0} C_{1}^{|\alpha|} \alpha_{i}^{\alpha_{i} d_{i}}
$$

for any $\alpha$, where $\|w, K\|_{\infty}$ means the maximum of $|w|$ in $K$.

As in $\$ 6$, let $\Omega$ be a domain in $R^{n}$ and let the boundary of $\Omega$ contain an open set $\omega(\neq \varnothing)$ in the plane $y=0$.

Now we can state our results.

THEOREM 9.1 (CF. [3]). Let $P(D)$ be the quasi-elliptic operator defined as above and take the boundary operators $D_{y}^{k}(j=1, \ldots, r)$ given above. Consider the boundary problem

$$
\begin{aligned}
P(D) u(x, y) & =f(x, y) \quad \text { in } \Omega, \\
D_{y}^{k} u(x, 0) & =0, \quad j=1, \ldots, r \text { on } \omega
\end{aligned}
$$

with $f \in G(\lambda q ; \Omega \cup \omega), \lambda q=\left(\lambda q_{1}, \ldots, \lambda q_{n}\right), \lambda \geqq 1$. Then any function $u \in \Phi_{1 \mathrm{oc}}^{m}(\Omega \cup \omega)$ satisfying (9.3) and (9.4) is a function in $G(\lambda q ; \Omega \cup \omega)$.

THEOREM 9.2. Let $P(D)$ be the same as in Theorem 9.1. Assume that a function $u \in C^{\infty}(\Omega \cup \omega)$ satisfies the following conditions:

(i) For each compact set $K$ in $\Omega \cup \omega$ there exist two constants $C_{0}, C_{1}$ such that

$$
\left\|P^{k}(D) u, K\right\|_{\infty} \leqq C_{0} C_{1}^{k}(m k)^{\lambda k} \quad(\lambda \geqq 1) .
$$

(ii) On the plane boundary $\omega$, it holds that

$$
D_{y}^{k_{1}} P^{k}(D) u(x, 0)=0, \quad k=0,1,2, \ldots,
$$

where $k_{j}, j=1, \ldots, r$ are the same as in Theorem 9.1. Then $u \in G(\lambda q ; \Omega \cup \omega)$.

REMARK. As a special case of Theorem 8.1 we see that any solution $u \in \Phi_{\mathrm{loc}}^{m}(\Omega \cup \omega)$ of the problem (9.3), (9.4) is infinitely differentiable on $\Omega \cup \omega$.

10. Proof of Theorem 9.1. To prove Theorems 9.1 and 9.2 we make use of the methods in [3], [2] and [8].

First we shall derive some preliminary lemmas by making use of the coerciveness inequalities proved in the foregoing paragraphs. 
We denote by $W$ the set of all functions $v \in C_{0}^{\infty}(\Omega \cup \omega)$ which satisfy boundary conditions (9.4).

Lemma 10.1. Let $P(D)$ be that in Theorem 9.1. Then for any $\varepsilon>0$ and for any $v \in W$ the following inequality holds:

$$
\sum_{\langle\alpha, q\rangle \leqq m}\left\|D^{\alpha} v\right\| \varepsilon^{\langle\alpha, q\rangle} \leqq C\left\{\varepsilon^{m}\|P(D) v\|+\left(1+\varepsilon^{m}\right)\|v\|\right\},
$$

where the constant $C$ is independent of $\varepsilon$ and $v \in W$.

Proof. First we see that, if $\langle\beta, q\rangle \leqq m$, then

$$
\left|\xi^{\beta^{\prime}} \eta^{\beta_{n}}\right| \leqq C\left(\sum_{1}^{n-1}\left|\xi_{j}\right|^{m_{j}}+|\eta|^{m_{n}}\right)^{\langle\beta, q\rangle / m}, \quad(\xi, \eta) \in R^{n},
$$

where the constant $C$ is independent of such $\beta$. In fact, replacing $\xi_{j}$ by $\xi_{j} t^{m / m_{j}}$, $j=1, \ldots, n-1$ and $\eta$ by $\eta t^{m / m_{n}}$ in (10.2) means multiplying both sides by $t$ if $t>0$. Hence (10.2) is valid. Next noting that $\langle\beta, q\rangle \mid m \leqq 1$ we can easily verify that, if $\langle\beta, q\rangle \leqq m$ then

$$
\left|\xi^{\beta^{\prime}} \eta^{\beta_{n}}\right| \varepsilon^{\langle\beta, q\rangle} \leqq C\left\{\varepsilon^{m}\left(\sum_{j=1}^{n-1}\left|\xi_{j}\right|^{m_{j}}+|\eta|^{m_{n}}\right)+1\right\}
$$

for another constant $C$ independent of $\operatorname{such} \beta$ and of $(\xi, \eta) \in R^{n}$. By the same way in the proof of Lemma 5.1, we get for any $\alpha,\langle\alpha, q\rangle \leqq m$ and for any $v \in W$

$$
\left\|D^{\alpha} v\right\| \varepsilon^{\langle\alpha, q\rangle} \leqq C\left\{\varepsilon^{m} \sum_{j=1}^{n}\left\|D_{j}^{m} j v\right\|+\|v\|\right\}
$$

Finally we apply the coerciveness estimate (3.8) and we get Lemma 10.1.

LEMMA 10.2 (CF. [3], [10]). For every compact set $K \subset\left(R_{+}^{n}\right)^{a}$ and for every $h, 0<h \leqq 1$, there are a function $\psi=\psi_{K, h}$ and constants $C_{\alpha}$ independent of $h$ such that $\psi \in C_{0}^{\infty}\left(K_{h}\right), \psi=1$ on $K$ and

$$
\left\|D^{\alpha} \psi\right\|_{\infty} \leqq C_{\alpha} h^{-\langle\alpha, q\rangle} \text { for every } \alpha,
$$

where $K_{h}=\left\{x \in\left(R_{+}^{n}\right)^{a} ; \operatorname{dis}(x, K) \leqq h\right\}$. It may be assumed that $D_{y}^{\dagger} \psi(x, 0)=0$, $i=1, \ldots, m_{n}$.

Now we introduce some notation. For convenience we assume that the plane boundary $\omega$ contains the origin $(0, \ldots, 0)$. We denote by $V$ the hemisphere $\left\{(x, y) ;|x|^{2}+y^{2}<R^{2}, y>0\right\}$ included in $\Omega$ and put $V_{-r}=\left\{(x, y) ;|x|^{2}+y^{2}<(R-r)^{2}\right.$, $y>0\}, 0<r<R \leqq 1$. We set for arbitrary $l \geqq 0$,

and

$$
\left\|D^{\beta} u ; l+\langle\beta, q\rangle, V\right\|=\sup _{0<r<R} r^{l+\langle\beta, q\rangle}\left\|D^{\beta} u, V_{-r}\right\|,
$$

$$
\begin{aligned}
\|u ; q, \mu ; l, V\|=\sup _{\beta \geqq 0 ; \beta_{n}=0} \prod_{i=1}^{n-1}\left(\frac{\mu_{i}}{\beta_{i}+1}\right)^{\beta_{i} q_{i}}\left\|D^{\beta} u ; l+\langle\beta, q\rangle, V\right\|, \\
\quad \mu=\left(\mu_{1}, \ldots, \mu_{n-1}\right), \mu_{i}>0,1 \leqq i \leqq n-1 .
\end{aligned}
$$


The following lemma is essential in our proofs of Theorem 9.1 and Theorem 9.2.

Lemma 10.3 (CF. [2]). Let $P(D)$ be the same as in Theorem 9.1, that is, $P(D)$ is quasi-elliptic of weight $q$ and of determined type $r$. Then

$$
\begin{aligned}
& \sum_{\langle\alpha, q\rangle \leqq m}\left\|D^{\alpha} u, V_{-r}\right\| \varepsilon^{\langle\alpha, q\rangle} \leqq C\left\{\varepsilon^{m}\left\|P(D) u, V_{-(r-\delta)}\right\|+\varepsilon^{m} \delta^{-m}\right. \\
&\left.\sum_{\langle\alpha, q\rangle<m} \delta^{\langle\alpha, q\rangle}\left\|D^{\alpha} u, V_{-(r-\delta)}\right\|+\left\|u, V_{-(r-\delta)}\right\|\right\}
\end{aligned}
$$

for any $\varepsilon>0$ and for any $u \in C^{\infty}(V)$ satisfying the boundary condition (9.4). The constant $C$ is independent of $r, \delta(0<\delta<r, 0<r<+\infty)$ and $u$.

Proof. Take $\psi=\psi_{v_{-r, \delta}}$ defined in Lemma 10.2. Then $\psi u$ also satisfies the boundary condition (9.4). Hence $\psi u$ satisfies the inequality (10.1). So

$$
\sum_{\langle\alpha, q\rangle \leqq m}\left\|D^{\alpha} u, V_{-r}\right\| \varepsilon^{\langle\alpha, q\rangle} \leqq C\left\{\varepsilon^{m}\|P(D)(\psi u)\|+\left(1+\varepsilon^{m}\right)\|\psi u\|\right\} .
$$

By the Leibniz formula we have

$$
P(D)(\psi u)=\psi P(D) u+\sum_{\langle\alpha, q\rangle \leqq m} \sum_{\beta_{i} \leqq \alpha_{i} ; \beta \neq 0} a_{\alpha} C_{\alpha, \beta} D^{\alpha-\beta} u D^{\beta} \psi,
$$

where $C_{\alpha, \beta}$ are appropriate constants. Hence we have

$$
\begin{aligned}
\|P(D)(\psi u)\| & \leqq C\left\|P(D) u, V_{-(r-\delta)}\right\|+C \sum_{\langle\alpha, q\rangle \leqq m} \sum_{\beta_{i} \leqq \alpha_{i} ; \beta \neq 0} \delta^{-\langle\beta, q\rangle}\left\|D^{\alpha-\beta} u, V_{-(r-\delta)}\right\|, \\
& \leqq C\left\|P(D) u, V_{-(r-\delta)}\right\|+C \sum_{\langle\gamma, q\rangle\langle m} \delta^{-m+\langle\gamma, q\rangle}\left\|D^{\gamma} u, V_{-(r-\delta)}\right\| .
\end{aligned}
$$

Thus the lemma is proved.

Now in (10.8) we put $\varepsilon=\chi \cdot t \cdot r, \delta=r t$ with sufficiently small $\chi, t(>0)$ determined later. Then (10.8) turns into

$$
\sum_{\langle\alpha, q\rangle \leqq m}\left\|D^{\alpha} u, V_{-r}\right\| \chi^{\langle\alpha, q\rangle}(t r)^{\langle\alpha, q\rangle} \leqq C\left\{\chi^{m}(t r)^{m}\left\|P(D) u, V_{-r(1-t)}\right\|\right.
$$

Multiplying both sides by $(\operatorname{tr})^{l}(l \geqq 0)$ we have

$$
\begin{array}{r}
+\chi^{m} \sum_{\langle\alpha, q\rangle\langle m}(r t)^{\langle\alpha, q\rangle}\left\|D^{\alpha} u, V_{-r(1-t)}\right\| \\
\left.+\left\|u, V_{-r(1-t)}\right\|\right\} .
\end{array}
$$

$$
\begin{aligned}
\sum_{\langle\alpha, q\rangle \leqq m}\left\|D^{\alpha} u, V_{-r}\right\| r^{l+\langle\alpha, q\rangle} \chi^{\langle\alpha, q\rangle} t^{\langle\alpha, q\rangle+l} & \\
\leqq C & \left\|P(D) u, V_{-r(1-t)}\right\|(r(1-t))^{l+m} \chi^{m}\left(\frac{t}{1-t}\right)^{l+m} \\
& +\chi^{m} \sum_{\langle\alpha, q\rangle<m}\left\|D^{\alpha} u, V_{-r(1-t)}\right\|(r(1-t))^{l+\langle\alpha, q\rangle}\left(\frac{t}{1-t}\right)^{l+\langle\alpha, q\rangle} \\
& \left.+\left\|u, V_{-r(1-t)}\right\|(r(1-t))^{l}\left(\frac{t}{1-t}\right)^{l}\right\} .
\end{aligned}
$$


Hence we get by (10.6)

$$
\begin{aligned}
& \sum_{\langle\alpha, q\rangle \leqq m}\left\|D^{\alpha} u ; l+\langle\alpha, q\rangle, V\right\| \chi^{\langle\alpha, q\rangle} t^{l+\langle\alpha, q\rangle} \\
& \leqq C\left\{\|P(D) u ; l+m, V\|\left(\frac{t}{1-t}\right)^{l+m} \cdot \chi^{m}+\chi^{m}\right. \\
& \left.\quad \sum_{\langle\alpha, q\rangle\langle m}\left\|D^{\alpha} u ; l+\langle\alpha, q\rangle, V\right\|\left(\frac{t}{1-t}\right)^{l+\langle\alpha, q\rangle}+\|u ; l, V\|\left(\frac{t}{1-t}\right)^{l}\right\} .
\end{aligned}
$$

Now assume $0<t \leqq 1 /(l+m)$. Then there is a constant $c>0$ such that

$$
(t /(1-t))^{l+\langle\alpha, q\rangle} \leqq t^{l+\langle\alpha, q\rangle} e^{c}
$$

for any $\alpha$ satisfying $\langle\alpha, q\rangle \leqq m$. Taking $\chi$ sufficiently small here we get, for another constant $C$,

$$
\sum_{\langle\alpha, q\rangle \leqq m}\left\|D^{\alpha} u ; l+\langle\alpha, q\rangle, V\right\| t^{\langle\alpha, q\rangle} \leqq C\left\{\|P u ; l+m, V\| t^{m}+\|u ; l, V\|\right\}
$$

with any $t$ such that $0<t \leqq 1 /(l+m)$.

We note that in Lemma 10.3 the terms $\varepsilon^{\langle\alpha, q\rangle}$ and $\delta^{\langle\alpha, q\rangle}$ can be replaced by $\varepsilon^{\langle\alpha, \lambda q\rangle}$ and $\delta^{\langle\alpha, \lambda q\rangle}$ with any $\lambda \geqq 1$ respectively. Thus we can obtain the following lemma.

LEMma 10.4. There exists a constant $C$ such that

$$
\sum_{\langle\alpha, q\rangle \leqq m}\left\|D^{\alpha} u ; l+\langle\alpha, \lambda q\rangle, V\right\| t^{\langle\alpha, \lambda q\rangle} \leqq C\left\{\|P(D) u ; l+m, V\| t^{m \lambda}+\|u ; l, V\|\right\}
$$

for all $u \in C(\Omega \cup \omega)$ satisfying the condition (9.4), provided that $0<t \leqq$ $1 /(l+m), \lambda \geqq 1$.

By making use of these lemmas we can prove Theorems 9.1 and 9.2. For simplicity we consider the case $\lambda=1$. It will be convenient to use the notation:

$$
\begin{gathered}
\left(D^{\beta} P(D) u\right)_{t}=t^{\langle\beta, q\rangle+m} D^{\beta} P(D) u, \quad\left(D^{\beta} u\right)_{t}=t^{\langle\beta, q\rangle} D^{\beta} u ; \\
B_{0}\left(D^{\beta} u\right)=\left\|\left(D^{\beta} u\right)_{t} ; l+\langle\beta, q\rangle, V\right\|\left({ }^{3}\right), \\
B_{i+1}(u)=\max _{\langle\beta, q\rangle \leqq m ; \beta_{n}=0} B_{i}\left(D^{\beta} u\right), \quad i \geqq 0 ; \\
B_{0}\left(D^{\beta} P u\right)=\left\|\left(D^{\beta} P u\right)_{t} ; l+\langle\beta, q\rangle+m, V\right\|, \\
B_{i+1}(P u)=\max _{\langle\beta, q\rangle \leqq m ; \beta_{n}=0} B_{i}\left(D^{\beta} P u\right), \quad i \geqq 0 ; \\
\|u ; q, \mu ; l, V\|=\sup _{\beta \leqq 0 ; \beta_{n}=0} \prod_{i=1}^{n-1}\left(\frac{\mu_{i}}{\beta_{i}}\right)^{\beta_{i} q_{i}}\left\|D^{\beta} u ; l+\langle\beta, q\rangle, V\right\| .
\end{gathered}
$$

$$
\|P u ; q, \mu ; l, V\|=\sup \prod_{i=1}^{n-1}\left(\frac{\mu_{i}}{\beta_{i}}\right)^{\beta_{i} a_{i}}\left\|D^{\beta} p u ; l+m+\langle\beta, q\rangle, V\right\| .
$$

( $\left.{ }^{3}\right)$ The $B_{\mathfrak{l}}$ are functions of $t$ also. 
LEMMA 10.5. There is a constant $C>1$ such that

$$
C^{-j} B_{j}(u) \leqq \max \left\{\max _{1 \leqq k \leqq j} C^{k-j} B_{j-k}(P u), B_{0}(u)\right\}
$$

for $j=1,2, \ldots$ and for all $u \in C^{\infty}(V)$ satisfying (9.4), provided that $0<t$ $\leqq 1 /(l+j m)$.

Proof. We see that $(10.10)$ means

$$
B_{1}(u) \leqq \max \left\{C B_{0}(P u), C B_{0}(u)\right\}
$$

with some positive constant $C$. The inequality $(10.15)$ shows that $(10.14)$ is true when $j=1$ and $0<t \leqq 1 /(l+m)$. If we replace $u$ by $D^{\beta} u$ in (10.8), multiply both sides by $\varepsilon^{\langle\beta, q\rangle}$, and then proceed as in the proof of Lemma 10.4, we find that

$$
B_{2}(u) \leqq \max \left\{C B_{1}(P u), C B_{1}(u)\right\},
$$

provided that $0<t \leqq 1 /(l+2 m)$. Again by (10.15) we obtain

$$
B_{2}(u) \leqq \max \left\{C B_{1}(P u), C^{2} B_{0}(P u), C^{2} B_{0}(u)\right\},
$$

provided that $0<t \leqq 1 /(l+2 m)$. Proceeding in this way, we can prove (10.14) for all $j$.

LEMMA 10.6. Let $B_{0}$ be defined by (10.12), (10.13) with $t_{j}=1 /(l+j m)$ for $l$ and $j$ fixed. Then there are constants $c<1$ and $C_{1}$, independent of $j$, such that

$$
C_{1}^{-1}\|u ; q, c \mu ; l, V\| \leqq C^{-j} B_{j}\left(P u, t_{j}\right)+B_{0}(u)
$$

and

$$
C^{-j} B_{j}\left(P u, t_{j}\right) \leqq\|P u ; q, \mu ; l, V\|,
$$

where $\mu=\left(C^{1 / q_{1}}, \ldots, C^{1 / q_{n-1}}\right)$.

Proof. Put $N=\|P u ; q, \mu ; l, V\|$, where $\mu=\left(C^{1 / q_{1}}, \ldots, C^{1 / q_{n-1}}\right)$, and suppose that $t=1 /(l+j m)$. Then

$$
\begin{aligned}
\max C^{-j} B_{j}(P u) & \leqq \max _{\langle\beta, q\rangle \leqq j m ; \beta_{n}=0} C^{-|\beta|}\left(\frac{1}{l+j m}\right)^{m+\langle\beta, q\rangle}\left\|D^{\beta} P u ; l+m+\langle\beta, q\rangle, V\right\| \\
& \leqq \max _{\langle\beta, q\rangle \leqq m ; \beta_{n}=0} C^{-|\beta|}\left(\frac{1}{l+j m}\right)^{m+\langle\beta, q\rangle} \prod_{i=1}^{n-1}\left(\frac{\beta_{i}}{\mu_{i}}\right)^{\beta_{i} q_{i}} N \\
& \leqq \max _{\langle\beta, q\rangle \leqq m ; \beta_{n}=0} \prod_{i=1}^{n-1}\left(\frac{\beta_{i}}{l+j m}\right)^{\beta_{i} q_{i}}\left(\frac{1}{l+j m}\right)^{m} N \leqq N .
\end{aligned}
$$

This proves (10.18). 
Next, with the same $t, \mu$ as above we have, for $c$ determined later,

$$
\begin{aligned}
C^{-j} B_{j}(u) \geqq & \max _{(j-1) m \leqq\langle\beta, q\rangle \leqq j m} C^{-j}\left(\frac{1}{l+j m}\right)^{\langle\beta, q\rangle}\left\|D^{\beta} u ; l+\langle\beta, q\rangle, V\right\| \\
\geqq & \max _{(j-1) m \leqq\langle\beta, q\rangle \leqq j m} C^{-j}\left(\frac{1}{l+j m}\right)^{\langle\beta, q\rangle} \prod_{i=1}^{n-1}\left(\frac{\beta_{i}}{c \mu_{i}}\right)^{\beta_{i} q_{i}} \prod_{i=1}^{n-1}\left(\frac{c \mu_{i}}{\beta_{i}}\right)^{\beta_{i} q_{i}} \\
& \cdot\left\|D^{\beta} u ; l+\langle\beta, q\rangle, V\right\| \\
\geqq & C^{\prime} \max _{(j-1) m \leqq\langle\beta, q\rangle \leqq j m} \prod_{i=1}^{n-1}\left[\frac{\beta_{i}}{c(l+j m)}\right]^{\beta_{i} q_{i}} \prod_{i=1}^{n-1}\left(\frac{\mu_{i}}{\beta_{i}}\right)^{\beta_{i} q_{i}}\left\|D^{\beta} u ; l+\langle\beta, q\rangle, V\right\|,
\end{aligned}
$$

where $C^{\prime}$ and $C_{0}$ are constants independent of $j$. Put

$$
K=\prod_{i=1}^{n-1}\left[\frac{\beta_{i}}{c(l+j m)}\right]^{\beta_{i} q_{i}}, \quad(j-1) m \leqq\langle\beta, q\rangle \leqq j m .
$$

Then

$$
\left(C^{\prime} K\right)^{-1}=\left(C^{\prime}\right)^{-1} \prod_{i=1}^{n-1}\left[\frac{c(l+j m)}{\beta_{i}+1}\right]^{\beta_{i} q_{i}} \leqq C_{1} c^{-\langle\beta, q\rangle}, \quad(j-1) m \leqq\langle\beta, q\rangle \leqq j m,
$$

is finite if $c$ is sufficiently small. This proves (10.17).

Finally we obtain the following estimate:

$$
\|u ; q, c \mu ; l, V\| \leqq C\{\|P u ; q, \mu ; l, V\|+\|u ; l, V\|\},
$$

for all $u \in C^{\infty}(V)$ satisfying (9.4). In the same way we can get the estimate of the type

$$
\left\|D^{\alpha} u ; q, c \mu ; l+\langle\alpha, q\rangle, V\right\| \leqq C\{\|P u ; q, \mu ; l, V\|+\|u ; l, V\|\}
$$

for any $\alpha$ such that $\langle\alpha, q\rangle \leqq m$.

Now we can complete the proof of Theorem 9.1. Let $f(x, y)$ be in $G(q, \Omega \cup \omega)$. Then for any hemisphere $K=\left\{(x, y) ;|x|^{2}+y^{2} \leqq r, y \geqq 0\right\} \subset V$, there are constants $C_{0}, C_{1}$ such that

$$
\left\|D^{\alpha} f, K\right\| \leqq C_{0} C_{1}^{|\alpha|}|\alpha|^{\langle\alpha, q\rangle}
$$

for all $\alpha$.

By the inequality (10.20) we have for new constants $C_{0}, C_{1}$

$$
\left\|D_{x}^{\beta} D^{\alpha} u, K\right\| \leqq C_{0} C_{1}^{|\beta|}|\beta|^{\langle\beta, q\rangle}
$$

for any $\beta\left(\beta_{n}=0\right)$ and $\alpha(\langle\alpha, q\rangle \leqq m)$. We may assume that the corresponding constants in (10.21) and (10.22) are the same.

The equation $P(D) u=f$ can be written in the form

$$
D_{y}^{m_{n}} u=f-\sum_{\langle\alpha, q\rangle \leqq m ; \alpha_{n}<m_{n}} a_{\alpha} D^{\alpha} u .
$$

We note that $\langle\alpha, q\rangle \leqq m$ and $\alpha_{n}=m_{n}$ imply $\alpha_{i}=0,(i=1, \ldots, n-1)$, and that for 
any positive integer $k\left(\leqq m_{n}\right),\langle\alpha, q\rangle \leqq m$ and $\alpha_{n}=m_{n}-k$ imply $\sum_{i=1}^{n-1} \alpha_{i} q_{i} \leqq k q_{n}$. Differentiating (10.23) with respect to $x$-variables and applying (10.21) and (10.22), we have

$$
\left\|D_{x}^{\beta} D_{y}^{m_{n}} u, K\right\| \leqq C_{0} C_{1}^{|\beta|}|\beta|^{\langle\beta, q\rangle}+B C_{0} C_{1}^{|\beta|}|\beta|^{\langle\beta, q\rangle},
$$

where we put $B=1+\sum_{\alpha}\left|a_{\alpha}\right|$. Again differentiating (10.23) we have

$$
\begin{aligned}
D_{x}^{\beta} D_{y}^{m_{n}+1} u= & D_{x}^{\beta} D_{y} f-\sum_{\langle\alpha, q\rangle \leqq m_{;} \alpha_{n}=m_{n}-1} a_{\alpha} D_{x}^{\beta} D_{x}^{\alpha^{\prime}} D_{y}^{m_{n}} u \\
& +\sum_{\langle\alpha, q\rangle \leqq} \sum_{m ; \alpha_{n} \leqq m_{n}-2} a_{\alpha} D_{x}^{\beta}\left(D_{x}^{\alpha^{\prime}} D_{y^{n}}^{\alpha_{n}+1} u\right)
\end{aligned}
$$

where we put $a_{\alpha}=0$ when $\alpha_{n}<0$. Applying (10.24) and Lemma 10.1 we have

$$
\begin{aligned}
\left\|D_{x}^{\beta} D_{y}^{m_{n}+1} u, K\right\| \leqq & C_{0} C_{1}^{|\beta|+1}(|\beta|+1)^{\langle\beta, q\rangle+q_{n}} \\
& +B(B+1) C_{0} C_{1}^{|\beta|+q_{n}}\left(|\beta|+q_{n}\right)^{\langle\beta, q\rangle+q_{n}} \\
& +B C_{0} C_{1}^{|\beta|+q_{n}+m}\left(|\beta|+q_{n}+m\right)^{\langle\beta, q\rangle+q_{n}+m} .
\end{aligned}
$$

Repeating the procedure we can obtain, by a simple induction argument on $k$,

$$
\begin{aligned}
\left\|D_{x}^{\beta} D_{y}^{m+k} u, K\right\| \leqq(B+1)^{k+1} C_{0} C_{1}^{|\beta|+k q_{n}+m}\left(|\beta|+k q_{n}+m\right)^{\langle\beta, q\rangle+k q_{n}+m}, \\
k=1,2, \ldots
\end{aligned}
$$

Hence we can take new constants $C_{0}, C_{1}$ such that

$$
\left\|D_{x}^{\beta} D_{y}^{m_{n}+k} u, K\right\| \leqq C_{0} C_{1}^{|\beta|+m_{n}+k}\left(|\beta|+m_{n}+k\right)^{\langle\beta, q\rangle+\left(m_{n}+k\right) q_{n}}
$$

for any $\beta\left(\beta_{n}=0\right)$ and for any $k \geqq 0$.

Applying Sobolev's lemma to the inequality (10.27) we obtain Theozem 9.1.

11. Proof of Theorem 9.2. From the estimate (10.10) we easily obtain

$$
\begin{aligned}
& \max _{\langle\alpha, q\rangle \leqq m ;\langle\beta, q\rangle \leqq k m ; \beta_{n}=0}\left\|D^{\beta+\alpha} u ; l+\langle\beta+\alpha, \lambda q\rangle, V\right\| t^{\langle\beta+\alpha, \lambda q\rangle} \\
& \leqq C^{k} \max _{0 \leqq j \leqq k}\left\|P^{j} u ; l+j m, V\right\| t^{\lambda j m}
\end{aligned}
$$

for all $u \in C^{\infty}(\Omega \cup \omega)$ satisfying (9.6), provided that $0<t \leqq 1 /(l+k m), k=0,1,2, \ldots$

In a quite similar manner to that used in the proof of Lemma 10.6 we obtain the estimate of the form

(11.2) $\max _{\langle\alpha, q\rangle \leqq m}\left\|D^{\alpha} u ; \lambda q, \mu ; l+\langle\alpha, \lambda q\rangle, V\right\| \leqq C \sup _{k}\left(\frac{\mu}{m k}\right)^{m \lambda k}\left\|P^{k} u ; l+\lambda k m, V\right\|$, where

$$
\left\|D^{\alpha} u ; \lambda q, \mu ; l+\langle\alpha, \lambda q\rangle, V\right\|=\sup _{\beta \geq 0 ; \beta_{n}=0} \prod_{i=1}^{n-1}\left(\frac{\mu_{i}}{\beta_{i}}\right)^{\beta_{i} q_{i}}\left\|D^{\beta+\alpha} u ; l+\beta+\langle\alpha, \lambda q\rangle, V\right\| .
$$

The same argument as in the end of the proof of Theorem 9.1 completes the proof of Theorem 9.2. 
Remark. The conclusion of Theorems 9.1 and 9.2 can be extended to operators with variable coefficients. The proof can be obtained by a quite similar argument to the proof of Theorem 9.1 and Theorem 9.2 (cf. [2], [8]).

Added in Proof. The conclusion of Theorems 9.1 and 9.2 can be extended to the general quasi-elliptic boundary problems defined in $\$ 3$. Details will be given in a future publication.

\section{REFERENCES}

1. S. Agmon, A. Douglis, and L. Nirenberg, Estimates near the boundary for solutions of elliptic partial differential equations satisfying general boundary conditions. I, Comm. Pure Appl. Math. 12 (1959), 623-727.

2. A. Cavallucci, Sulle proprietà differenziali delle soluzioni delle equazioni quasi-ellitiche, Ann. Mat. Pura Appl. 67 (1965), 143-168.

3. J. Friberg, Estimates for partially hypo-elliptic differential operators, Medd. Lunds Univ. Mat. Sem. 17 (1963), 96 pp.

4. L. Hörmander, On the regularity of solutions of boundary problems, Acta Math. 99 (1958), 225-264.

5. — Linear partial differential operators, Springer, Berlin, 1963.

6. Y. Kametaka, Sur la régularité au bord des solutions des équations paraboliques, Proc. Japan Acad. 41 (1965), 109-113.

7. J. Lions and E. Magenes, Espaces du type de Gevrey et problèmes aux limites pour diverses classes d'équations d'évolution, Ann. Mat. Pura Appl. 72 (1966), 343-394.

8. T. Matsuzawa, Regularity at the boundary for solutions of hypo-elliptic equations, Osaka J. Math. 3 (1966), 313-334.

9. S. Mizohata, Une remarque sur les opérateurs différentiels hypoelliptiques et partiellement hypoelliptiques, J. Math. Kyoto Univ. 1 (1962), 411-423.

10. C. Morrey and L. Nirenberg, On the analyticity of the solutions of linear elliptic systems of partial differential equations, Comm. Pure Appl. Math. 10 (1957), 271-290.

11. J. Peetre, On estimating the solutions of hypo-elliptic differential equations near the plane boundary, Math. Scand. 9 (1961), 337-351.

12. M. Schechter, On the dominance of partial differential operators. II, Ann. Scuola Norm. Sup. Pisa 18 (1964), 255-282.

Mathematical Institute, NAGOYA UNIVERSITY 